\title{
Paper-based microfluidics for rapid diagnostics and drug delivery
}

\author{
Kang Mao ${ }^{\mathrm{a}}$, Xiaocui Min ${ }^{\mathrm{b}}$, Hua Zhang ${ }^{\mathrm{a}^{*}}$, Kuankuan Zhang ${ }^{\mathrm{a}}$, Haorui Cao ${ }^{\mathrm{a}}$, Yongkun Guo ${ }^{\mathrm{a}}$, and \\ Zhugen Yang ${ }^{\mathrm{c}^{*}}$ \\ ${ }^{a}$ State Key Laboratory of Environmental Geochemistry, Institute of Geochemistry, Chinese \\ Academy of Sciences, Guiyang, 550002, China \\ ${ }^{b}$ Guangzhou Huali Science and Technology Vocational College, Guangzhou, 511325, China \\ ${ }^{c}$ Cranfield Water Science Institute, Cranfield University, Cranfield, MK43 OAL, United
}

Kingdom

\section{Content}

Abstract

Keywords

1. Introduction

2. Paper microfluidics and paper-based analytical devices

3. Paper-based analytical device integrated with LAMP

3.1. Brief introduction of LAMP

3.2. Recent application of LAMP-based paper devices

3.2.1. Rapid diagnosis of infectious diseases

3.2.2. Diagnosis of foodborne pathogens

3.2.3. Veterinary diagnosis

3.2.4. Plant diagnosis

3.2.5. Environmental and public health assessment

4. Paper microfluidics for drug delivery

5. Conclusion and perspectives

Acknowledgement

References 


\begin{abstract}
Paper is a common material that is promising for constructing microfluidic chips (lab-on-a-paper) for diagnostics and drug delivery for biomedical applications. In the past decade, extensive research on paper-based microfluidics has accumulated a large number of scientific publications in the fields of biomedical diagnosis, food safety, environmental health, drug screening and delivery. This review focuses on the recent progress on paper-based microfluidic technology with an emphasis on the design, optimization and application of the technology platform, in particular for medical diagnostics and drug delivery. Novel advances have concentrated on engineering paper devices for point-of-care (POC) diagnostics, which could be integrated with nucleic acid-based tests and isothermal amplification experiments, enabling rapid sample-to-answer assays for field testing. Among the isothermal amplification experiments, loop-mediated isothermal amplification (LAMP), an extremely sensitive nucleic acid test, specifically identifies ultralow concentrations of DNA/RNA from practical samples for diagnosing diseases. We thus mainly focus on the paper device-based LAMP assay for the rapid infectious disease diagnosis, foodborne pathogen analysis, veterinary diagnosis, plant diagnosis, and environmental public health evaluation. We also outlined progress on paper microfluidic devices for drug delivery. The paper concludes with a discussion on the challenges of this technology and our insights into how to advance science and technology towards the development of fully functional paper devices in diagnostics and drug delivery.
\end{abstract}

Keywords: Paper-based microfluidics, LAMP, diagnostic, drug delivery

\title{
1. Introduction
}

Disease identification and treatment are always major focuses in biomedical research $[1$, 2]. Microfluidic technology plays a significant role in the evolution of biomedical research [3-6]. The rapid diagnosis and drug delivery using microfluidics are rapidly growing fields with a large number of biomedical and clinical diagnoses, forensic analyses, food safety assurances and environmental detections [7-10]. There are various microfluidic devices based on silicon [11], glass [12], polymers [13-15], and paper material [16, 17]. Among these materials, paper-based microfluidics, as one of the most attractive devices, has been widely reported since Whitesides's group reported the first paper microfluidic device [17-19]. Generally, paper microfluidics using cellulosic paper can process 0.1 to $100 \mu \mathrm{L}$ of liquid by millimetre-scale fluid channels [17]. Cellulosic paper is a ubiquitous, lightweight, biodegradable, and inexpensive material that could become a natural platform for microfluidics [16]. Although paper-based microfluidic devices lack a high mechanical robustness, they hold some other merits compared with traditional microfluidic materials [20]: the hydrophilicity and porosity of cellulose generate flow through capillary action when there is a lack of power supply or external pump, and patterning techniques can create well-defined channels using conventional fabrication approaches [17]. Paper-based microfluidic devices are particularly popular in environments requiring a low cost, easy operation, and rapid analysis [20]. Although many diagnostic applications have been used in developing countries due to limited resources and expertise, there are also many applications in developed countries [21]. These advantages promote the development of this field as paper-based microfluidics is used in portable devices for diagnostics and drug delivery [15, 22]. 
The purpose of early diagnosis is to enable patients to receive early treatment to cure them, which in turn promotes the innovation and development of more efficient diagnosis methods [23]. As an effective diagnostic technology, nucleic acid detection technology plays a significant role in an accurate diagnosis [24-27]. First, as a common genetic material, nucleic acids hold unique sequences and quantities in different species of organisms; second, nucleic acids are easy to detect because all sequences, including DNA and RNA, are composed of four basic nucleotides. Third, currently, numerous nucleic acid sequences can be easily synthesized in vitro at a reasonable cost in a very short time. Fourth, nucleic acid detection is routine because nucleic acids are easy to operate, purify, label and sequence. Finally, with the rapid development of nucleic acid sequencing techniques, nucleic acid detection could meet the requirements of clinical applications [28]. Therefore, these accurate and sensitive nucleic acid detection results provide efficient evidence for a medical diagnosis. Currently, nucleic acid-based techniques have been widely applied in monitoring infectious diseases, diagnosing cardiovascular disease, and screening for cancer [29].

Since the amount of nucleic acids extracted from samples is very small, the amplification protocol holds a significant role in the subsequent detection [25-27]. The rapid development of nucleic acid amplification technology has experienced the most significant progress, and it has a significant impact on biomedical applications due to improvements in diagnostic accuracy, selectivity and sensitivity [6]. Currently, the most widely used nucleic acid amplification technique is polymerase chain reaction (PCR) [30]. Although PCR is a good choice for labs with expensive proprietary equipment and experienced technicians, the necessary thermal cycling increases the difficulties and costs in the point-of-care application [31]. Isothermal methods are promising techniques to replace PCR, and these operate at a constant temperature for the amplification of nucleic acids. The isothermal process makes the amplification method in POC diagnostic equipment possible and helps to develop disease detection methods in resource-limited areas [32]. Moreover, different isothermal approaches could combine with the amplification reaction in a multichannel readout [28, 33]. Isothermal amplification reactions can be classified into three types depending on the reaction kinetics, including the linear, exponential and cascade amplification [26]. Among these isothermal amplification reactions, exponential amplification has a higher efficiency and detection sensitivity [26]. Many exponential amplification methods have been proposed, and these include LAMP [34], strand displacement amplification (SDA) [35], and rolling circle amplification (RCA) [36]. The LAMP technique has attracted much more attention for nucleic acid determination due to its stability, sensitivity, and specificity.

LAMP has become the most widely used isothermal amplification method due to its simple instrumentation and excellent specificity compared with other isothermal amplification methods [34, 37]. According to the process of primer annealing followed by autocycling strand displacement at elevated and constant temperatures, LAMP uses six specific primers in the first stage and four primers during the amplification, elongation, and recycling steps. LAMP has many applications in clinical detection, veterinary diagnosis, food safety analysis, and environmental health analysis $[1,38,39]$. However, biological samples are complex in composition and low in target nucleic acid amounts, requiring arduous nucleic acid extraction and subsequent amplification before analysis. To overcome these limitations, integrated paper-based devices as efficient tools could incorporate nucleic acid extraction, nucleic acid 
amplification and visual detection or quantification through cell phones, lateral flow tests, and even the naked eye [40-42]. In addition, adding the sample into the microfluidic environment of the device reduces the risk of sample contamination and minimizes the sample volume needed for detection $[28,33]$. The diagnostic technologies made significant progress when the LAMP assay was coupled with paper microfluidics, as this brought a method for diagnostic molecular biology to areas with limited resources [43]. In the past several years, rich and diverse achievements have been made in the field of LAM-based paper microfluidics devices [44-46].

We briefly reviewed the progress of paper-based microfluidic devices for point-of-care diagnostics and drug delivery. The research of fundamental paper microfluidics about imbibition has been well reported (listed in Tab. S1) and has not been discussed in our review [47-49]. In the first part, we simply introduce paper microfluidic and paper analytical devices; then, we review the paper-based device integrated with isothermal amplification. Third, we review the diagnostic technologies performed on paper-based microfluidic devices coupled with LAMP, the main application in the rapid diagnosis of infectious diseases, analysis of food safety, evaluation of agriculture and farm substances, and evaluation of environmental public health. Some experts have tried to make full use of paper microfluidics in drug delivery, but there are only several reports thus far. Therefore, we simply introduce the paper microfluidics used in drug screening and delivery in the last part.

\section{Paper microfluidics and paper-based analytical devices}

The occurrence of paper microfluidics technology has the potential to replace standard microfluidic substrates with paper [19]. Paper is a cheap and ubiquitous material in the world. Being burnable, it reduces the risk of contamination. Moreover, paper wicks aqueous fluids, and this wicking makes passive transport of fluids on paper possible without a pump or power supply. Herein, paper has been used as an analytical platform for decades, and thus paper-based analytical devices could make full use of existing analytical techniques [50].

Apart from the characteristics mentioned above, paper holds some other merits for miniaturization and portable analytical devices: (i) paper is easy to stack, store, and transport because it is thin and lightweight and has different thicknesses [51]; (ii) paper is compatible with biological samples because it is usually composed of cellulose or cellulose polymers [52]; (iii) paper could contain all kinds of functional groups for binding to DNA, small molecules and proteins through chemical modifications [53]; (iv) white paper has a strong contrast with coloured substrates, making it a perfect medium in colorimetric analyses; (v) paper-based analysis equipment could be incinerated after use because paper is flammable; (vi) flexible paper could easily fabricate paper devices because it is compatible with many printing technologies [50]; and (vii) thin fibrous paper sheets have many highly engineered forms with many properties [51]. Using filter paper as an example, it can isolate suspended solids in samples and remove red blood cells from blood before analysis due to the pores on the paper have a certain diameter. Paper with conductive carbon/metal fibres has conductivity or a magnetic response, and paper containing biodegradable polymers could make paper-based analysis equipment biocompatible [50].

Patterning technology is a critical factor in promoting the development of paper analytical device-based POC assays [54]. Some scientists invented a micropatterning technique for paper microfluidics by wax printing and showed that channels along with liquids that are 
forced to flow could be designed in the same manner as standard microfluidics [54]. With this patterning technology, paper-based microfluidics have occurred as a novel research field of microfluidic devices. All these advantages have promoted paper devices towards the applications of clinical diagnosis, food safety analysis and environmental health evaluation [55-58].

\section{Paper-based analytical device integrated with LAMP}

\subsection{Brief introduction of LAMP}

LAMP is a mature technology, which is discussed in many publications (listed in Tab. S1) $[26,28,33,34,59-63]$. However, when we introduced the application of paper device in this field, it is mainly based on LAMP-based paper device. In order to make readers understand well about LAMP-based paper microfluidics for rapid diagnostics, we need to briefly introduce the basic principle of LAMP and its characteristics.
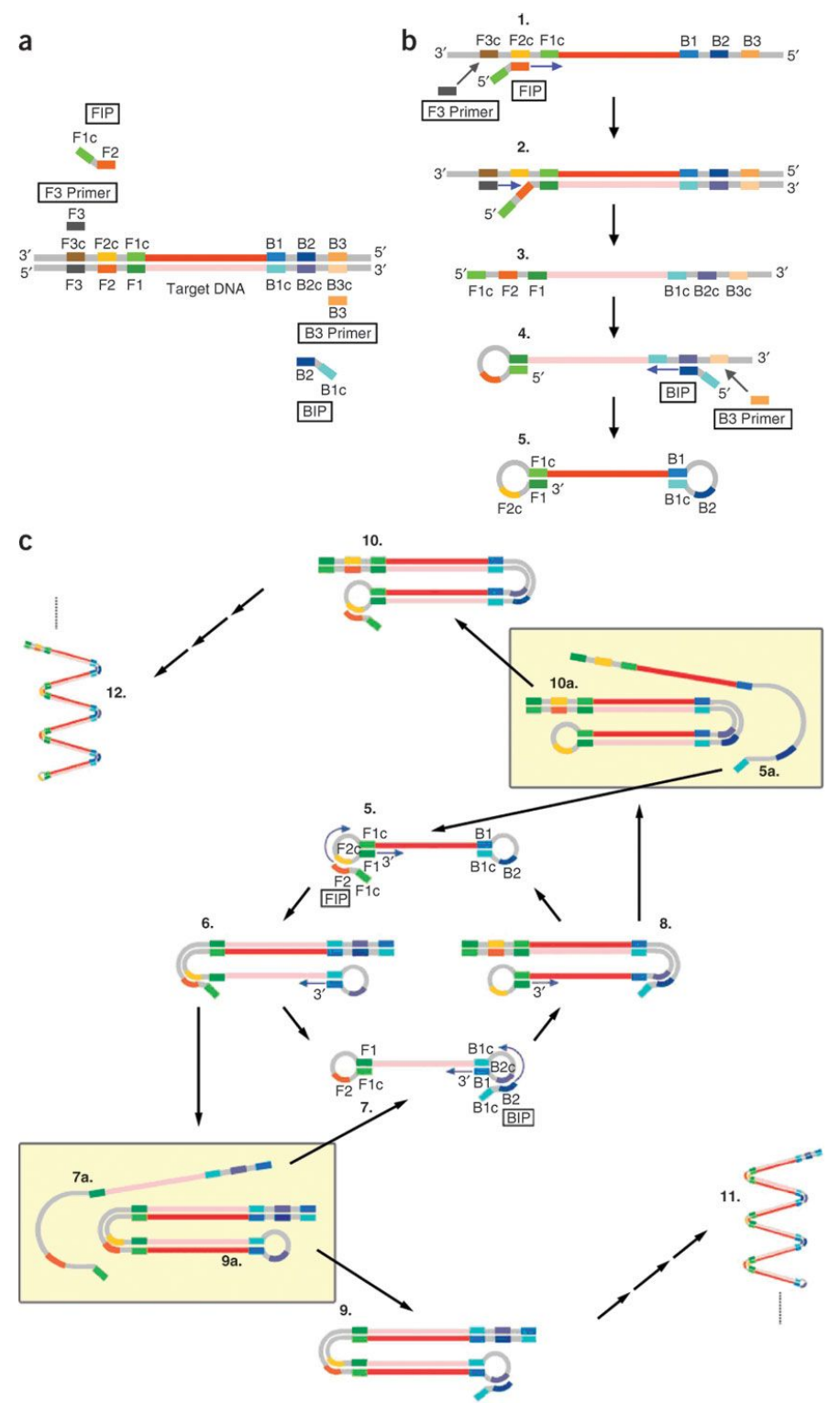

Fig. 1. Principle of the loop-mediated isothermal amplification method (Reprinted with permission from ref [63]). 
LAMP could obtain excellent specificity by four or six targeted specific primers, including the forward inner primer (FIP), the backward inner primer (BIP), and two outer primers (F3 and B3), to recognize six distinct sites flanking the amplified nucleic acid in a one-step reaction (see Fig. 1A). Usually, researchers use two other auxiliary loop primers to help make a LAMP reaction perform well. As shown in Fig. 1, FIP and BIP play major roles in LAMP, and each contains two functional nucleic acids (one is for the priming extension in the first step, and the other is for the self-priming in the second step), corresponding to the sequences (sense and antisense) of the target double-stranded DNA (dsDNA). DNA polymerases with strand displacement activity catalyse the reaction of LAMP and include two steps: the initial structure generation stage and the cyclic amplification stage [34, 63]. Four primers are used in the first step, while only two inner primers are used in the second step. Briefly, ssDNA is released by strand displacement nucleic acid synthesis primed by F3 and then acts as the template for DNA synthesis primed by both BIP and B3, generating a stem-loop nucleic acid (Fig. 1B). After the initiation by one inner primer complementary to the loop on the product, the cycling amplification reaction is continued by the alternation of each inner primer, resulting in the geometric accumulation of $10^{9}$ copies of the target sequence in less than an hour (Fig. 1C). The final products are stem-loop nucleic acids with different inverted target repeats and cauliflower-like structures with multiple loops; the acquired amplicons can be analysed through end-point or even real-time detection [64].

LAMP is extremely sensitive and fast for detecting nucleic acids, and this suggested to be an ideal characteristic for POC diagnostics; more importantly, it does not require thermal cycling like PCR; thus, it can even be used in low-resource settings [63]. LAMP methods are more specific and sensitive due to using four to six specific primers that bind certain sites of the template nucleic acid sequence, while two primers are required by PCR. LAMP amplifies nucleic acids using the nucleic acid polymerase enzyme at temperatures between 60 and $65{ }^{\circ} \mathrm{C}$. LAMP not only produces more than 50 -fold amplicons but also amplifies middle to long strands of nucleic acid templates, ranging from 130 to $300 \mathrm{bp}$. Herein, LAMP is an appropriate method for a variety of pathogenic DNA amplifications. Moreover, polymerase inhibitors from clinical samples have no effect on the amplification reaction, making LAMP a wonderful candidate for point-of-care diagnostics. Thus, LAMP could amplify certain targets in complicated samples even with substances usually inhibiting PCR, and these include haemoglobin in blood and some food ingredients. Another key advantage of LAMP is that pyrophosphate occurs when the reaction occurs, allowing the reaction to be visualized by the naked eye. LAMP is efficient and widely employed for the detection of infectious diseases, food pathogens, single nucleotide polymorphisms, and gene mutations [1, 62].

\subsection{Recent application of LAMP-based paper devices}

There is an urgent need to develop miniaturized and portable devices for point-of-care nucleic acid assays. The crucial factor for the amplification of target nucleic acids is to increase the sensitivity, making the feasibility of detecting single cells and few copies of target DNA/RNA. Some merits of the amplification process miniaturization and integration with sample pretreatment and assay on the same device include preventing contamination, using less expensive reagents and having fewer manual steps. LAMP has become a novel technology for the DNA/RNA amplification method for POC testing due to the simplicity of 
its hardware and its high specificity and sensitivity. Paper-based microfluidics, as one of the most attractive devices, has received wide attention due to its unique characteristics, including inexpensive, simplicity, and integration function. Therefore, paper microfluidics coupled to LAMP provide the opportunity for rapid molecular diagnosis technology for real clinical applications and have been widely utilized in different fields, including the diagnosis of infectious diseases, analysis of food safety, diagnosis of veterinary and plant diseases, and assessment of environmental and public health concerns (listed in Tab. 1).

Tab. 1. LAMP-based paper microfluidics for rapid diagnosis of various targets.

\begin{tabular}{|c|c|c|c|c|c|}
\hline Target & $\begin{array}{l}\text { Nuclei } \\
\text { c acids }\end{array}$ & LOD & $\begin{array}{l}\text { Time } \\
(\mathrm{min}) \\
\end{array}$ & $\begin{array}{l}\text { Detection } \\
\text { method }\end{array}$ & Ref. \\
\hline Pneumonia & DNA & $20 \mathrm{fg} \mu \mathrm{L}^{-1}$ & 75 & Smartphone & [44] \\
\hline Malaria & DNA & $10^{-5} \mathrm{IU} \mathrm{mL}^{-1}$ & $<50$ & Lateral flow test & [21] \\
\hline Malaria & DNA & 5 parasites $\mathrm{mL}^{-1}$ & 45 & Fluorescence & [65] \\
\hline $\begin{array}{l}\text { Paediatric } \\
\text { gastroenteritis }\end{array}$ & DNA & $\begin{array}{l}1000 \text { copies } \\
\mathrm{mL}^{-1}\end{array}$ & 30 & Naked eye & [45] \\
\hline Zika virus & RNA & 1 сору $\mu \mathrm{L}^{-1}$ & 45 & Smartphone & [66] \\
\hline Amphimeriasis & DNA & - & - & Fluorescence & [67] \\
\hline $\begin{array}{l}\text { Listeria } \\
\text { monocytogenes }\end{array}$ & DNA & 100 copies & 60 & Fluorescence & [68] \\
\hline HIV & RNA & $10 \mathrm{fg} \mu \mathrm{L}^{-1}$ & 60 & Electrochemical & [69] \\
\hline $\begin{array}{l}\text { Aflatoxigenic } \\
\text { Aspergillus sp. }\end{array}$ & DNA & 100aflR copies & 40 & Colorimetry & [70] \\
\hline Meningitis & DNA & 3 copies & 45 & Fluorescence & [71] \\
\hline HPV & DNA & $10^{4}$ copies & 60 & Lateral flow strip & [72] \\
\hline E. coli & DNA & $25 \mathrm{CFU} \mu \mathrm{L}^{-1}$ & 40 & Colorimetry & [22] \\
\hline E. coli & DNA & $0.13 \mathrm{ng} \mu \mathrm{L}^{-1}$ & 70 & Fluorescence & [46] \\
\hline E. coli & DNA & 5 cells & 70 & UV/camera & [79] \\
\hline E. coli & DNA & $10 \mathrm{CFU} \mathrm{mL}^{-1}$ & 60 & Lateral flow test & [80] \\
\hline E. coli & DNA & $100 \mathrm{CFU} \mathrm{mL} \mathrm{m}^{-1}$ & 65 & Naked eye & [81] \\
\hline E. coli & DNA & 250 copies $\mu \mathrm{L}^{-1}$ & 40 & Visual & [82] \\
\hline E. coli & DNA & $100 \mathrm{CFU} \mathrm{mL} \mathrm{L}^{-1}$ & 40 & Fluorescence & [83] \\
\hline Salmonella & DNA & $25 \mathrm{CFU} \mu \mathrm{L}^{-1}$ & 40 & Colorimetry & [22] \\
\hline Salmonella & DNA & $170 \mathrm{CFU} \mathrm{mL} \mathrm{mL}^{-1}$ & 70 & Fluorescence & [46] \\
\hline Salmonella & DNA & $100 \mathrm{CFU} \mathrm{mL} \mathrm{m}^{-1}$ & 65 & Naked eye & [81] \\
\hline Salmonella & DNA & - & - & Visual & [82] \\
\hline $\begin{array}{l}\text { Cochlodinium } \\
\text { polykrikoides }\end{array}$ & DNA & $0.12 \mathrm{ng} \mu \mathrm{L}^{-1}$ & 70 & Fluorescence & [46] \\
\hline $\begin{array}{l}\text { Staphylococcus } \\
\text { aureus }\end{array}$ & DNA & 21.5 copies $\mu \mathrm{L}^{-1}$ & 60 & Fluorescence & [84] \\
\hline $\begin{array}{l}\text { Vibrio } \\
\text { parahaemolyticus }\end{array}$ & DNA & 20.9 copies $\mu \mathrm{L}^{-1}$ & 60 & Fluorescence & [84] \\
\hline $\begin{array}{l}\text { Mycobacterium } \\
\text { smegmatis }\end{array}$ & DNA & $100 \mathrm{CFU} \mathrm{mL}^{-1}$ & 40 & Fluorescence & [83] \\
\hline Sus scrofa & DNA & $1 \mathrm{pg} \mu \mathrm{L}^{-1}$ & 10 & Colorimetry & [85] \\
\hline Bacillus subtilis & DNA & $10 \mathrm{pg} \mu \mathrm{L}^{-1}$ & 18.2 & Colorimetry & [85] \\
\hline PRRSV & RNA & 100 copies & 35 & Colorimetry & [86] \\
\hline $\begin{array}{l}\text { Tritrichomonas } \\
\text { foetus }\end{array}$ & DNA & 5 cells $\mathrm{mL}^{-1}$ & 90 & Visual & [87] \\
\hline BoHV-1 & DNA & $<1 \mathrm{pg}$ & 60 & Fluorescence & [88] \\
\hline Brucella & DNA & $<1 \mathrm{pg}$ & 60 & Fluorescence & [88] \\
\hline Leptospira & DNA & $<1 \mathrm{pg}$ & 60 & Fluorescence & [88] \\
\hline
\end{tabular}




\begin{tabular}{llllll}
\hline $\begin{array}{l}\text { Genetically } \\
\text { modified crops }\end{array}$ & DNA & $0.03 \%$ & 45 & Lateral flow test & {$[91]$} \\
$\begin{array}{l}\text { Insect-resistant } \\
\text { genetically }\end{array}$ & DNA & $0.50 \%$ & 35 & Color & {$[93]$} \\
modified rice & & & & & \\
$\begin{array}{l}\text { Genetic biomarker } \\
\text { Gene mutation }\end{array}$ & DNA & 40 copies & 45 & Lateral flow test & {$[98]$} \\
$\begin{array}{l}\text { Antibiotic-resistan } \\
\text { t gene }\end{array}$ & DNA & 3 cells & 90 & Lateral flow test & {$[73]$} \\
\hline
\end{tabular}

\subsubsection{Rapid diagnosis of infectious diseases}

In recent years, the spread of infectious diseases has threatened the health and life of people worldwide. Rapid and accurate diagnosis technology to detect relevant pathogens is critical to prevent infectious diseases. LAMP showed its large potential when it was first used to monitor a virus for an acute respiratory syndrome [23]. Currently, much more attention has been paid to LAMP, and it has become a popular method for the rapid diagnosis of infectious diseases [1, 62]. In addition, the combination of paper microfluidics and LAMP greatly accelerated this further application in the field of infectious disease diagnosis. To date, the LAMP-based paper device has been widely applied for the rapid detection of nucleic acids possessing human- and non-human-carried bacteria and viruses for disease diagnosis. In this section, we reviewed LAMP-based paper devices for the detection of different diseases, such as pneumonia [44], malaria[21, 65], paediatric gastroenteritis [45], Zika virus [66], amphimeriasis [67], Listeria monocytogenes[68], human immunodeficiency virus (HIV) [69], aflatoxigenic Aspergillus sp.[70], meningitis[71], and human papilloma virus (HPV) [72]. In addition to diagnosing infectious diseases, other clinical diagnostics using LAMP-based paper devices have also been reported, and these include detecting gene mutations [73] and diagnosing antibiotic-resistant genes [74].

Malaria: Malaria is one of the main reasons for morbidity and mortality worldwide. A rapid, cheap and specific diagnosis depending on the detecting of nucleic acids is increasingly important in the early diagnosis and treatment of infectious diseases. Xu et al developed a whole-blood method based on paper origami for the diagnosis of malaria [65]. They demonstrated the integrated steps of DNA extraction, LAMP reaction and array-based fluorescence analysis by paper-folding technology for analysing multiplex microbial species in the blood (Fig. 2A). A cheap flashlight was used to show the final nucleic acid amplicons with the naked eye, providing a point-of-care analysis within 45 min using human blood from punctured fingers. To verify the accuracy of their method, they identified three Plasmodium species by analysing 80 samples of patients and comparing results to results from standard PCR by blind sample manipulation. In comparison with commercial LAMP kits, their method had two merits: LAMP-based paper origami could recognize P. falciparum and P. vivax infections and accurately identified non-vivax and non-falciparum infections while also providing a pan-genus analysis as a single test. This means that the management and treatment needed for these two different infections were different.

Here, we also described an innovative paper-based microfluidics that enabled multiplexed nucleic acid-based diagnostics for malaria in an underserved rural area in Uganda with low resources [21]. Paper device technology combined the processing steps of vertical flow samples by using paper folding for sample pretreatment, a LAMP reaction and a lateral flow assay, and the integration into a visualization system. Fig. 2B (a) shows the integration of the 
paper/plastic lateral flow assay system employed for the reaction and determination. Attached to this device was a foldable paper strip to extract and process nucleic acids. The unfolded paper strip consisted of dark regions that were printed by hydrophobic wax and unpatterned regions that had direct sample flow. The right side fold is the waste panel, the sample panel to its left demonstrates where the sample was loaded, and the distributing channel is where DNA was eluted for nucleic acid testing. The left fold sits on top of the three LAMP testing chambers (arrow 5). Pink shading on the lateral flow strip was due to the coloured detection particles stored here. The scale bar is related to the whole device. Fig. 2B (b) shows a graphical scheme of the plastic microfluidic device and highlights its main characteristics.

The test was performed in schools in rural Uganda, and every test was finished within 50 min, which was faster than PCR. This highly sensitive and specific method was used to detect malaria in $98 \%$ of infected individuals in a double-blind study, enabling the detection of malaria in patients' whole blood samples. The LAMP-based paper microfluidics could sensitively analyse multiplexed targets and gave a high-quality, fast and precise diagnosis for malaria compared to PCR. This method not only showed that efficient and cheap nucleic acid-based biosensors could be employed in less developed areas in the field but also highlighted the challenges of developing and implementing new diagnostic techniques without laboratories.

(A)

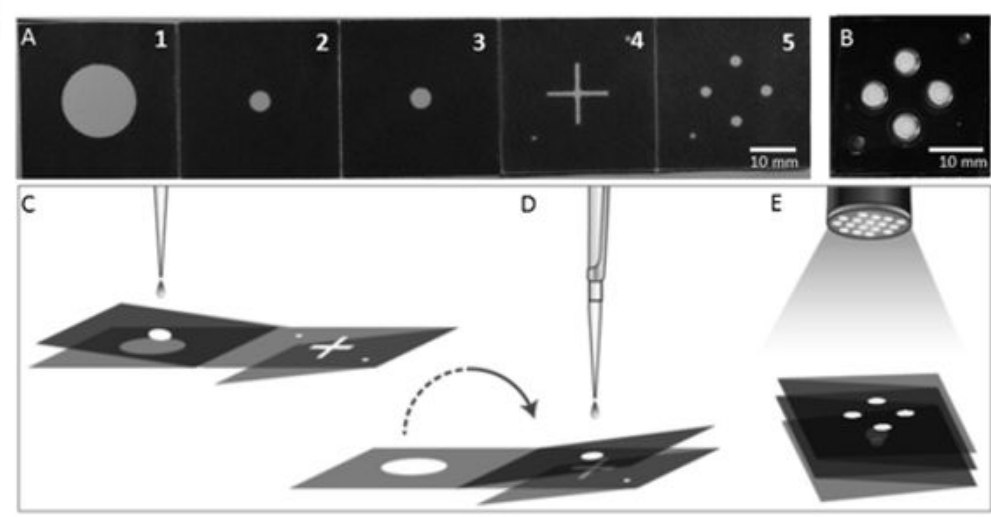

(B)

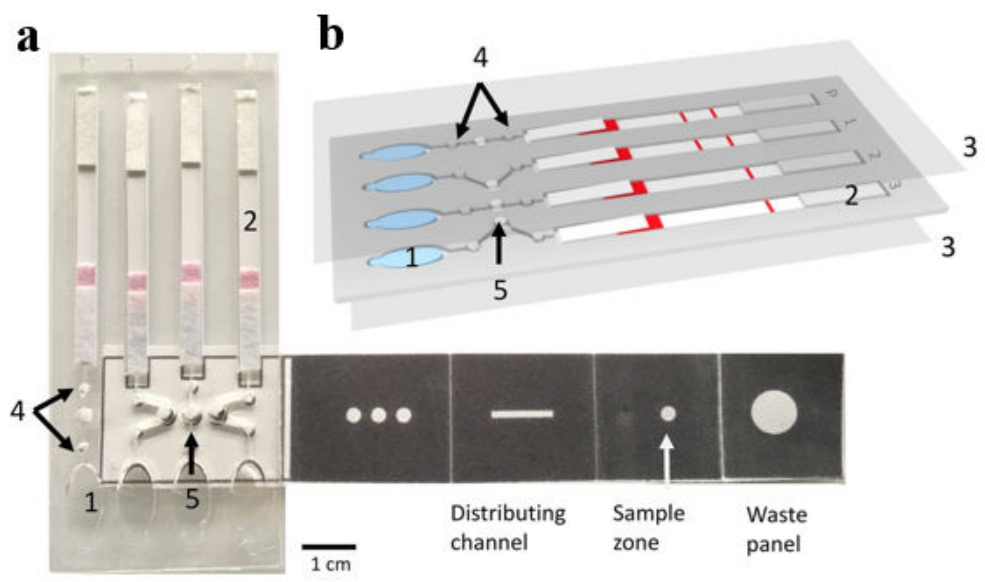

Fig. 2. (A) Paper-origami-based multiplexed malaria diagnostics from whole blood (Reprinted with permission from ref [65] ); (B) Paper-based microfluidic device for multiplex LAMP-based detection of malaria in blood (Reprinted with permission from ref [21] ). 
Zika virus: The current Zika outbreak began in Brazil in 2015 and has since spread to more than 70 countries in South and Central America, Africa and Asia [75]. A major problem with Zika virus infection is the increased risk of congenital central nervous system abnormalities, including microcephaly, especially in the early stages of pregnancy [76]. As sexual transmission has been discovered apart from mosquito-based infection, accurate and rapid diagnosis of Zika virus infection is of great significance not only for pregnant women with serious birth defects but also for their male partners [77]. Additionally, the recent Zika virus outbreak accelerated the demand for on-site testing that was rapid while providing satisfactory sensitivity and specificity. Herein, Kaarj et al developed a novel Zika virus assay using a reverse transcription LAMP-based paper device and a cell phone [66]. Fig. 3A shows that the paper device was designed with SolidWorks and was printed by wax on grade 4 cellulose paper. Printed wax patterns were melted and vertically flown through the depth of paper by placing it on a hot plate. The bright area represents the hydrophilic area where liquid could flow, and the dark wax area represents the hydrophobic barrier. Fig. 3B shows that Zika virus-spiked samples were loaded onto the loading area and spontaneously flowed through the channel via capillary action. Contaminants in the sample matrices were filtered during this process, while target nucleic acids flowed through. Fig. 3C shows that the circular end-detection area of the device was cut, and the reverse transcription-LAMP mixture was added to it. This cut-out paper was placed on a hot plate at $68{ }^{\circ} \mathrm{C}$ for up to $40 \mathrm{~min}$ for the amplification reaction. Successful amplification reactions were detected with the colorimetric method and quantified by cell phone imaging (Fig. 3C). This novel paper device could also be applied for the on-site identification of other transmitted pathogens.

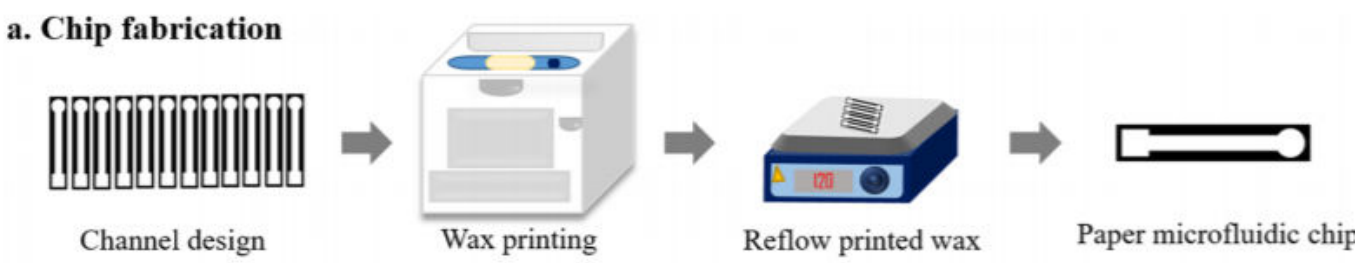

\section{b. Microfluidic filtration}

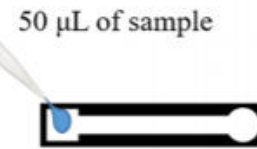

c. RT-LAMP on chip

$15 \mu \mathrm{L}$ of RT-LAMP reaction mixture

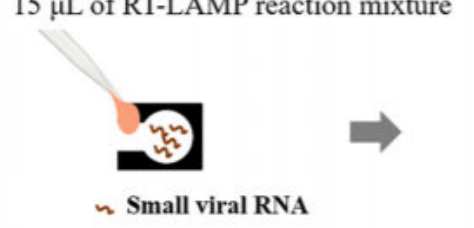

n Small viral RNA

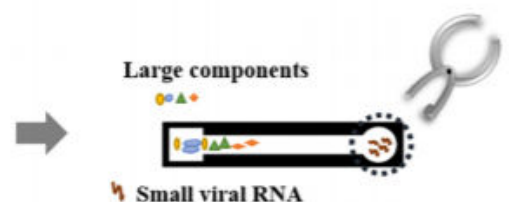

h Small viral RNA

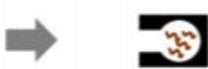

Small viral RNA
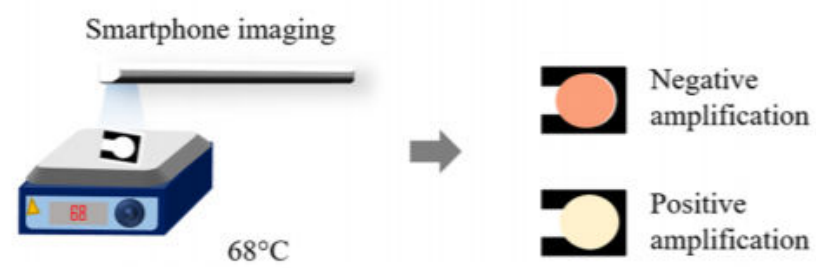

Fig. 3. Schematic of paper microfluidic reverse transcription-LAMP assay (Reprinted with permission from ref [66]). 
Human immunodeficiency virus (HIV): HIV is the main cause of death due to a single source of infection, and the development of convenient HIV detection methods is increasingly important. Although common immunoassays for anti-HIV antibodies enabled rapid HIV infection diagnosis, these methods could not be quantified and were not sufficiently sensitive for HIV determination during seroconversion. Moreover, when individuals infected by HIV are most contagious, they do not have sufficient anti-HIV antibody for a diagnosis [78]. Therefore, rapid and sensitive POC diagnostics are very important for early HIV diagnosis and treatment. Safavieh et al used a paper electrode composed of a silver nanocomposite modified by graphene for the electrochemical analysis of HIV [69]. Here, with the combination of LAMP, they presented cellulose paper and flexible plastic chips with printed silver electrodes modified by graphene as a POC analytical device for the determination of HIV. This inexpensive method achieved the sensitivity based on guidelines by the World Health Organization and could be applied to detect antiretroviral treatment on-site.

Pneumonia diagnosis: Community-acquired pneumonia (CAP) can cause death in children under the age of five worldwide. Mycoplasma pneumoniae (M. pneumoniae) and Streptococcus pneumoniae (S. pneumoniae) are the most common pathogens that can cause CAP. It is necessary to develop a rapid and inexpensive early diagnostic method with satisfactory sensitivity and selectivity. Herein, Wang et al reported a universal paper microfluidic device combined with LAMP for duplex detection of $S$. pneumoniae and $M$. pneumoniae on-site [44]. This approach included polymer/paper microfluidics and a miniaturization device. The pathogenic nucleic acids were extracted by magnetic beads on a chip within $15 \mathrm{~min}$ and were amplified by LAMP. The miniaturization device could heat the microfluidics, take a picture and transmit the experimental result to a cell phone. They randomly selected 63 oropharyngeal swabs and bronchoalveolar lavage fluid specimens from children for analysis using the developed and standard methods to evaluate the clinical accuracy, sensitivity and specificity. The experimental results showed that this inexpensive analytical tool has an opportunity to be implemented as a POC device for analysing different kinds of pathogens, especially in resource-limited areas.

Meningitis: Given that bacterial meningitis causes high morbidity and mortality worldwide, especially in low-income areas, a rapid and cost-effective analytical technique for the early monitoring of meningitis on-site is urgently needed. Dou et al [71] incorporated the LAMP technique into a paper device for an on-site assay for detecting Neisseria meningitidis ( $N$. meningitidis), which is the main bacteria that causes meningitis. Compared with paper-free devices, the integration of paper into the microfluidics for LAMP required a much shorter time within $45 \mathrm{~min}$ and achieved the sensitivity of a single bacterium by on-site detection. This inexpensive paper device provided an ultrasensitive analytical method for the detection of $N$. meningitidis without instrumentation in a limited-resource environment, and this showed a promising alternative for a variety of infectious diseases, especially in developing countries.

Apart from the pathogens causing infectious diseases, Ye et al designed a novel diagnosis method by integrating instrument-free nucleic acid extraction ( $5 \mathrm{~min}$ ) and amplification (25 min) processes into a paper device for the POC diagnosis of rotavirus A, which was the main cause of paediatric gastroenteritis [45]. Rodriguez et al integrated nucleic acid extraction, amplification, and determination into a paper device for HPV diagnosis from clinical samples 
[72]. All these analytical tools were composed of paper material, making them cost-effective and disposable, and all target nucleic acid sequences were amplified by LAMP, making them highly sensitive and selective. Therefore, LAMP-based paper devices offer the opportunity for the on-site diagnosis of infectious diseases.

\subsubsection{Diagnosis of foodborne pathogens}

Food safety, especially pathogen prevention, is one of the most significant aspects of public health. Therefore, foodborne pathogen contamination prevention and food quality control are very important. The sensitive and specific approach to monitor foodborne pathogens is to analyse certain nucleic acids that comprise bacterial cells; by disinfecting and lysing, DNA can be extracted, amplified, and detected using amplified sequences. Therefore, nucleic acid assays play a significant role in microbial pathogen determination for food safety. Recently, LAMP-based paper devices have been widely applied in food safety and foodborne pathogen detection. These pathogens including Escherichia coli (E. coli) [22, 46, 79-83], Salmonella [22, 46, 81, 82], Cochlodinium polykrikoides [46], Staphylococcus aureus (SA) [84], Vibrio parahaemolyticus (VP) [84], food allergens [83], Sus scrofa (porcine) [85], and Bacillus subtilis [85] have also been detected using these devices. We introduced several examples of the different foodborne pathogen detection methods mentioned above. In particular, we highlighted a study from Lee's group because they made a great contribution to foodborne pathogen detection using a LAMP-based paper device [22, 46, 81, 82].

The foodborne pathogen $E$. coli is the main reason for foodborne disease outbreaks. A rapid analytical method for $E$. coli with a satisfactory sensitivity and specificity is needed. Detection of $E$. coli using a LAMP-based paper device has been widely reported. Connelly et al designed a "paper machine" for detecting E. coli [79]. They described a paper microfluidics method that integrates sample pretreatment and LAMP with endpoint detection by utilizing a hand-held ultraviolet source and smartphone. The prototype device integrated paper to enable fluid handling, and a multilayer structure allowed a central patterned paper strip to slide in and out of the fluidic channel, thus allowing the addition of a sample and other necessary reagents with minimal pipetting in a hand-held, disposable device intended for POC use. This device created a dynamic seal that prevented evaporation when the sample was incubated at $65{ }^{\circ} \mathrm{C}$ for $1 \mathrm{~h}$. This interval was sufficient to allow a LAMP reaction for the $E$. coli gene to proceed with an analytical sensitivity of 1 dsDNA target copy, and an LOD of 5 cells was acquired using the full integration of the paper machine by human plasma spiked with whole live $E$. coli cells. Choi et al also developed an integrated paper-based sample-to-answer biosensor for on-site E. coli testing [80].

Apart from the detection of a single pathogen (E. coli), the rapid duplex detection of different pathogens has also been reported. Naik et al successfully integrated a 30-minute one-step assay combining thermal lysis and LAMP from E. coli and M. smegmatis cells into a single reaction stage on a paper substrate [83]. E. coli and M. smegmatis could be completely disinfected on paper by heating at $60{ }^{\circ} \mathrm{C}$ for 5 and $15 \mathrm{~min}$, respectively, making this assay safe and suitable for the incorporation into diverse paper devices for field use. They overcame a big challenge of seamless integration of these steps on a paper substrate. This problem was even more complex for Mycobacteria, as its thick cell wall structure impedes lysis and requires careful optimization of enzymatic denaturation.

Many studies on other foodborne pathogen analyses using LAMP-based paper devices have 
been published. Roy et al developed a device for using colorimetric nucleic acid extracted from meat samples on a paper microchip using LAMP and crystal violet dye for detecting Sus scrofa (porcine) and Bacillus subtilis (bacteria) [85]. Here, they presented a paper microchip to detect and quantify nucleic acids using a colorimetric sensing modality. The extracted DNA from food samples of meat as well as microbial pathogens was amplified utilizing LAMP. The LAMP amplicons were then detected and quantified on a paper microchip fabricated in cellulose paper and a small wax chamber utilizing crystal violet dye. The affinity of the crystal violet dye is towards dsDNA, and a positive signal was identified by the colour changing from colourless to purple. Using this method, the detection of Sus scrofa and Bacillus subtilis DNA was possible at concentrations as low as $3.43 \times 10^{-1}$ and $2.2 \times$ $10^{3}$ copies $\mu \mathrm{L}^{-1}$, respectively.

Pang et al also developed a self-priming PDMS/paper hybrid microfluidic chip using a mixed-dye-loaded LAMP assay for detecting the multiplex foodborne pathogens, Staphylococcus aureus and Vibrio parahaemolyticus [84]. Compared to other similar detection devices, the paper device had an easier fabrication process, fewer operation steps, and a lower cost. Additionally, the reaction result, especially for the weak-positive reaction, could be judged more accurately and conveniently due to the use of mixed dye. Without pre-enrichment of bacteria in the food-contaminated sample, the LOD was $1000 \mathrm{CFU} \mathrm{mL} \mathrm{mL}^{-1}$ with high specificity. Overall, the reliable and excellent results demonstrated that the novel method had great potential to be applied in the wider range of pathogen detection or disease diagnosis, especially in some resource-limited areas.

Lee's group has performed substantial work in the foodborne pathogen detection and identification areas over the last two years. We introduced four highlight studies from Lee's group in this part. First, Trinh et al developed a rapid and eco-friendly LAMP-based paper device and on-chip fluorescence for multiplex detection of foodborne pathogens [46]. Fig. 4A shows that papers injected with LAMP reagents and certain primers were infused inside the multiple reaction chambers of the device. A solution containing the target pathogens was injected into the sample chamber, located in the centre of the microfluidic device, and evenly and simultaneously distributed to the reaction chambers via centrifugal force. Purified nucleic acids of E. coli, Salmonella spp., Staphylococcus aureus ( $S$. aureus), and Cochlodinium polykrikoides were amplified and detected on the microfluidic device, and the LODs of

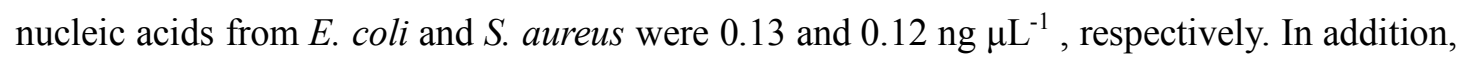
the potential of this microfluidic device for POC testing was further examined by incorporating an on-chip sample purification module and using a real sample milk spiked with Salmonella spp. The thermally lysed milk sample was filtered using polydopamine-coated paper embedded inside a sample chamber and seamlessly transported into the reaction chambers by centrifugal force for subsequent LAMP followed by direct on-chip detection inside the reaction chambers, in which fluorescent molecule-soaked papers were embedded. The limit of detection for Salmonella spp. was determined to be $1.7 \times$ $10^{2} \mathrm{CFU} \mathrm{mL} \mathrm{m}^{-1}$ using the microfluidic device. This selective and sensitive device was safe enough to monitor foodborne pathogens and other environmentally harmful microorganisms on site.

Trinh et al continued to construct a foldable LAMP-based paper device that integrated the DNA purification, amplification, and detection processes for fuchsin-based colour assays of 
multiple foodborne pathogens [81]. Fig. 4B shows that the device was composed of three parts: a sample zone, a reaction zone, and a detection zone. A sealing film attached to the sample, reaction, and detection zones acted as a bottom layer to allow the microfluidic device to be foldable. The detection zone was made up of paper strips attached to the sticky side of the sealing film, and fuchsin-stained lines were drawn on the paper strips. The target nucleic acid solution could be directly transferred from the sample chambers to the reaction chamber by folding. The fluid operation was easy to achieve, and there was no need to use large instruments, such as pumps or spinners, in this way. The detection zone was also folded to make the fuchsin-stained lines sufficiently soaked into the reaction chambers after the LAMP reaction. Nucleic acid sequences of Salmonella spp. and E. coli were purified from thermally lysed milk using paper coated with polydopamine, amplified by LAMP, and detected by the naked eye for less than $65 \mathrm{~min}$. These experimental results demonstrated that the paper microfluidic device had an important potential for analysing genes from a variety of foodborne pathogens in an environment with limited resources.

(A)

(B)
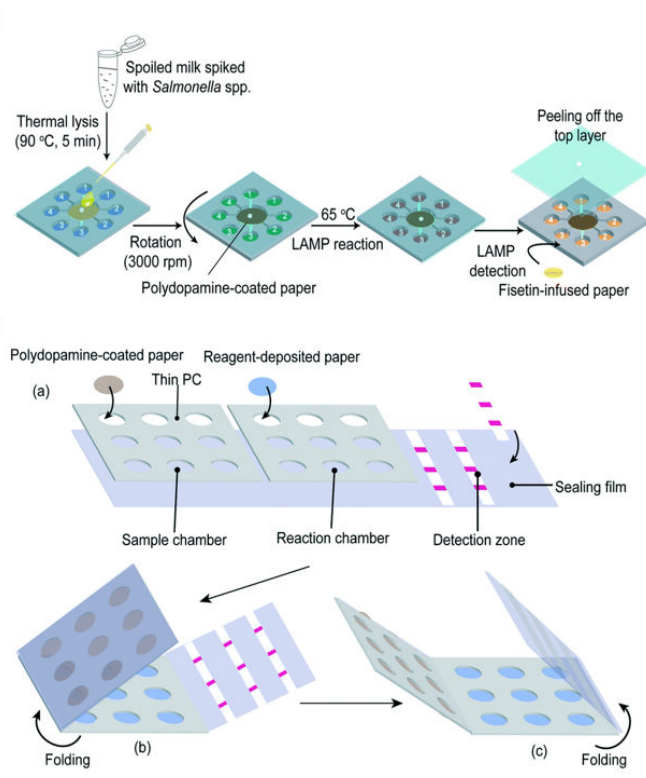

(C)

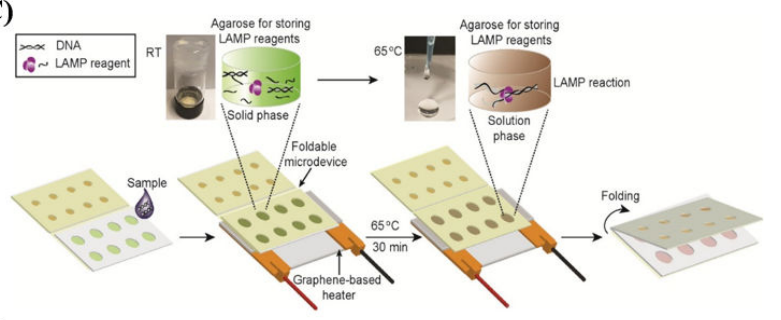

(D)

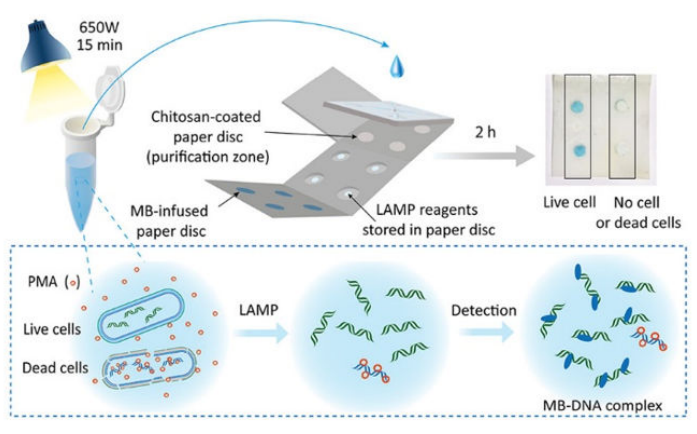

Fig. 4. (A) A rapid and eco-friendly isothermal amplification microfluidic device for the multiplex detection of foodborne pathogens (Reprinted with permission from ref [46]); (B) A foldable isothermal amplification microfluidic device for the fuchsin-based colorimetric detection of multiple foodborne pathogens (Reprinted with permission from ref [81]); (C) Fully integrated and foldable microfluidic device encapsulated with agarose for long-term storage that has potential for POC testing of multiplex foodborne pathogens (Reprinted with permission from ref [82]); (D) Paper-based all-in-one origami microfluidic device for nucleic acid amplification testing for rapid colorimetric identification of live cells for POC testing (Reprinted with permission from ref [22]).

For the rapid identification of live cells of foodborne pathogens, Lee's group [22] reported a novel method for live cell identification, depending on DNA amplification, using an origami paper device integrated with nucleic acid purification, LAMP, and point-of-care colorimetric assay (seen in Fig. 4D). First, the paper material was partially embossed to create 
microchannel networks and chambers. Subsequently, to stabilize the structures and provide fluid barriers, hydrophobic polydimethylsiloxane prepolymer was coated onto the embossed paper. The paper microfluidic device was composed of splitting, purifying, wicking, reacting, and alternatively folding dye pads to accomplish nucleic acid amplification tests. Propidium monoazide was applied to penetrate dead cells and form covalent bonds with nucleic acids of necrotic cells for the viability assay, which made the DNA amplification process only occur in live bacterial cells. Chitosan was utilized to electrostatically capture nucleic acids for purification. Methylene blue was added for the colorimetric assay of amplicons. This origami paper device has successfully been employed to detect multiple foodborne pathogens, such as E. coli and Salmonella spp. This amplification method was operated for $30 \mathrm{~min}$ followed by the execution of the colour detection for $10 \mathrm{~min}$, demonstrating this method has great potential for multiplexing and versatility for the on-site detection of pathogens.

For the long-term storage potential of paper devices, they continue to construct a foldable paper device encapsulated with 2-hydroxyethyl agarose for the integration of LAMP and subsequent colorimetric assay for detecting multiplex foodborne pathogens (seen in Fig. 4C) [82]. Fig. 4C demonstrates that the device comprised a reaction zone and a detection zone. Both zones are foldable because they were made of a thin polycarbonate film and sealed by an adhesive. For the long-term maintenance of reagent activity, the reaction and detection chambers deposited 2-hydroxyethyl agarose with LAMP reagents and silver nitrate, respectively. A heater-based thin graphene associated with a handheld battery was utilized to provide a constant temperature for amplification within $30 \mathrm{~min}$. Using the agarose, the reagents could maintain the reagent activity for at least 45 days. Before performing multiplex detections, the spiked juice was thermally lysed and purified with paper coated by polydopamine. This method was successful in the identification of Salmonella spp. and E. coli with high sensitivity and selectivity; promising convenient and rapid methods for foodborne pathogen assays have paved the way for the fabrication of miniaturization and visual public health evaluation platforms.

\subsubsection{Veterinary diagnosis}

The health of animals is a significant concern to ensure productive agricultural outputs. LAMP-based paper devices have also been applied in agriculture or on farms to detect transmitted infectious pathogens in animals, and these include porcine reproductive and respiratory syndrome virus (PRRSV) [86], Tritrichomonas foetus (T. foetus) [87], bovine herpes virus-1 (BoHV-1), Brucella and Leptospira [88] detection in animals [89].

Herdsmen on pastures in low- and middle-income countries usually have difficulty in the timely monitoring and even early diagnosis of sexually transmitted infectious pathogens due to the limited infrastructure for veterinary medicine. We fabricated a LAMP-based paper device for the multiplexed diagnosis of three bovine infectious reproductive diseases in semen samples and successfully applied it in rural India [88] (seen in Fig. 5). The detection signal was fluorescence, which could be collected either visually by a hand-held torch or by a smartphone. Nucleic acids from pathogens, including Brucella, Leptospira and BoHV-1, were extracted, amplified, and identified; target nucleic acids less than $1 \mathrm{pg}$ could be analysed. The paper device was verified by double-blind tests, and the LOD of the paper device for the three pathogens was also assessed as low as $1 \mathrm{TCID}_{50}$ for BoHV-1, $50 \mathrm{CFU}$ for Brucella, and $50 \mathrm{CFU}$ for Leptospira. The inexpensive and straightforward paper devices for the multiplex 
detection of infectious diseases demonstrated satisfactory specificity and sensitivity and have the ability to be applied to diagnosing other animal in resource-limited areas. Using the same strategy but for different targets, Oyhenart et al used LAMP technology and directly tested for the field detection of T. foetus in cattle genital fluid with paper strips [87].
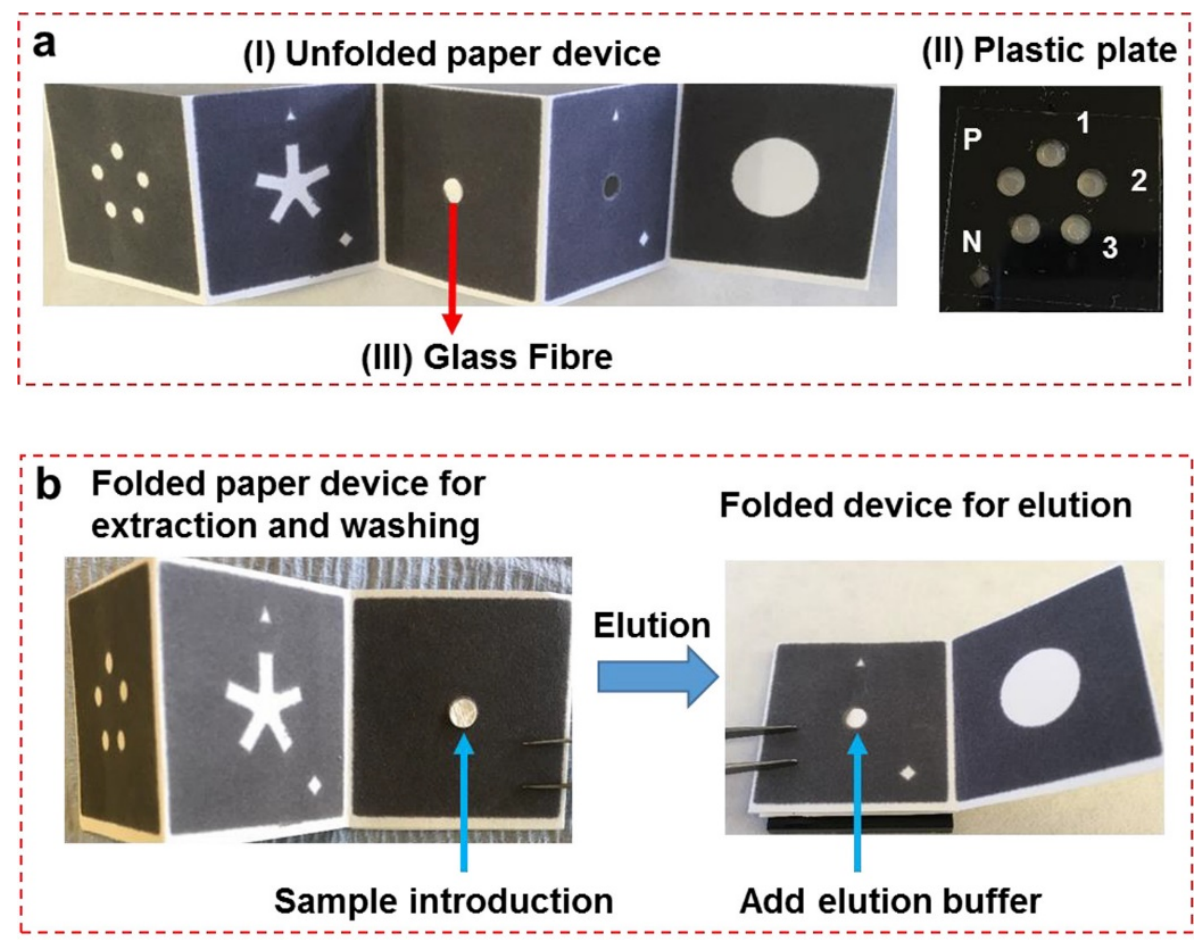

Detection after amplification

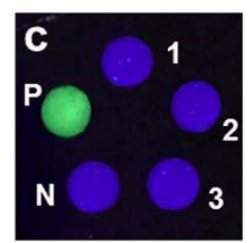

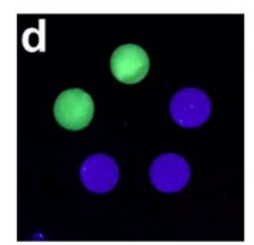

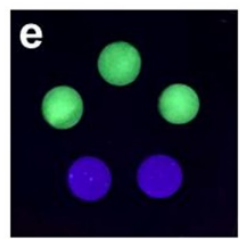

$3 \mathrm{~mm}$

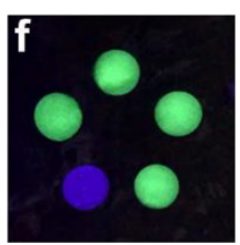

Fig. 5. Rapid veterinary diagnosis of bovine reproductive infectious diseases from semen using paper-origami DNA microfluidics (Reprinted with permission from ref [88] ).

\subsubsection{Plant diagnosis}

Genetically modified crops have increased by more than 100 million hectares worldwide [90-92]. However, some consumers expressed their concerns about genetically modified crops used for food. In response, laws required that genetically modified food must be labelled in many countries and regions. To efficiently manage the increased genetically modified events, Takabatake developed a rapid screening detection of genetically modified crops by LAMP with a lateral flow dipstick [91]. Single-stranded tag hybridization for a chromatographic printed-array strip system was used for the simultaneous identification of the mannose-6-phosphate isomerase gene, cauliflower mosaic virus $35 \mathrm{~S}$ promoter, a GA21-specific sequence, Pisum sativum ribulose 1,5-bisphosphate carboxylase terminator and a common sequence between the CrylAb and CrylAc genes. The special system was applicable to perform multiplex analyses. By using these rapid and useful diagnosis tools, the results are visible to the naked eye for screening and monitoring genetically modified crops 
without any expensive instruments. Zhang et al made full use of LAMP technology and designed instrument-free screening tools for the detection of insect-resistant genetically modified rice [93]. They successfully used this LAMP-based paper screening strategy for the identification of three popular insect-resistant genetically modified rice events in China for approximately half an hour.

\subsubsection{Environmental and public health assessment}

Wastewater-based epidemiology (WBE) is a novel tool for determining chemicals and illicit drug consumption in certain areas by analysing residues in wastewater [94, 95]. Wastewater contain much more efficient information on the health, lifestyle and contamination of an area. For example, Choi et al explored the relationships between the chemicals in wastewater and the social, demographic, and economic parameters of the respective populations in Australia [96]. Many biomarkers and pathogens of humans, including patients at hospitals, or animals can enter wastewater by faeces and urine. Pathogens in wastewater are toxic because they could lead to the outbreak of an epidemic disease. However, the risk of disease outbreak could be minimized if these pathogens could be identified at an early stage. Different from drug use trends, infectious diseases need to be assessed in a timely and real-time manner to determine whether there is a need for the containment of the disease carriers to certain areas to prevent epidemic disease outbreaks. Therefore, an identification device processed by unskilled personnel for the accurate and rapid detection of low concentrations of pathogens/biomarkers on-site is urgently needed. Therefore, we proposed the concept of a community sewage sensor to evaluate public health [97]. Emerging LAMP-based paper devices, as important sensing technology, would play an important role in the on-site analysis of biomarkers and pathogens in wastewater for public health due to the incomparable advantages mentioned above.

A LAMP-based lateral flow device was developed by our group to detect genetic biomarkers for WBE [98]. It was a point-of-care platform that could quantify the genetic biomarkers within communities via wastewater analysis. The assay was based on the LAMP of nucleic acid biomarkers and showed the ability of rapid quantification of human-specific mitochondrial DNA (mtDNA) from original wastewater. mtDNA was related to carcinogenesis, including gastric, breast and renal cancers, and acted as a model biomarker in this experiment. The functions of removing impurities, extracting and enriching nucleic acids were integrated into an inexpensive test-based lateral flow to enable a sample-to-answer technology. An LOD of 40 copies of mtDNA was achieved by detecting mtDNA for over seven consecutive days with minimal user intervention and within 45 min on site, providing a method to monitor public health from wastewater. LAMP-based paper devices were applied to monitor biomarkers of diseases and health and then to acquire real-time data, which showed the opportunity to evaluate the community health by examining wastewater-based epidemiology.

\section{Paper microfluidics for drug delivery}

The traditional drug delivery system is evenly distributed throughout the body and can rapidly degrade and excrete a drug across many biological barriers, leaving the drug in the pathological site. Controlled drug delivery is to release the drug to certain sites at the desired rate and time to improve the efficacy, pharmacokinetics and bioavailability of the drug while maintaining the minimal side effects. Due to some unique characteristics (precise and even 
multiple dosing, ideal and target-precise release, sustainable and controlled release, and slight side effects) of the recent microfluidic techniques, unprecedented opportunities exist to control drug delivery $[15,99]$. A drug could be efficiently delivered to the target site at a certain rate in a well-controlled way by microfluidics through the integration, implantation, localization, automation, and precise control of various parameters of the microfluidic device [10]. These features accordingly make reproducible, on-demand, and tunable drug delivery feasible. On-demand self-tuning dynamic drug delivery systems have shown great potential for personalized drug delivery [100]. The research field of conventional microfluidics used in drug delivery has been well elaborated previously [101-104] and was not discussed in this part. In this section, we will discuss the recent progress on paper-based microfluidic techniques for drug delivery systems, mainly including the fabrication of high-throughput screening of drugs, drug carriers and drug delivery systems.

Ding et al designed a microfluidic-enabled print-to-screen platform for the high-throughput screening of combinatorial chemotherapy [105]. Although the combination chemotherapy was applied as a standard method to treat cancer since the 1960s, the conventional drug combinations for the identification are usually associated with high operational costs, low-throughput screening, laborious and time-consuming procedures, and ethical concerns due to a large number of potential drug candidates. Herein, they presented a cost-effective and efficient microfluidic print-to-screen (P2S) platform, which integrated combinatorial screening with biomolecular printing for high-throughput screening of anticancer drug combinations. Microfluidic impact printing utilizing plug-and-play microfluidic cartridges was experimentally characterized with controllable droplet volume and accurate site. What's more, the combinatorial P2S assay was presented in a proof-of-concept biological experiment that could identify the positive hits among the whole drug combination library in a parallel and rapid way. This P2S platform had several unique merits, including automatic combinatorial printing, high-throughput parallel drug screening, modular disposable cartridge, and biocompatibility, which potentially improved the entire discovery cycle of potent drug combinations. Overall, this microfluidic print-to-screen platform offered a high-efficiency but low-cost method for large-scale and high-throughput combinatorial screening and could be used in drug cocktail discovery in various emerging applications.

Hong et al tried their best to design a high-throughput drug screening device and therefore developed a concentration gradient generator on a paper microfluidic device coupled with a cell culture microarray [106]. Inspired by the paper platform for 3D cell culture, a paper device containing a drug concentration gradient was fabricated for investigating cell response to drugs based on high throughput analysis (seen in Fig. 6). This drug gradient generator was applicable for generating concentration gradients of doxorubicin (DOX) as the model drug. HeLa cells encapsulated in collagen hydrogel were incubated in the device reservoirs for evaluation of the cell viability based on the controlled delivery of DOX spatially. These results showed that drug diffusion through the paper fibers created a gradient of drug concentration, which have a significant on cell viability. The drug screening device has the ability to be used in field of drug discovery and diagnostics to simultaneously monitor drugs under various gradient concentrations. 


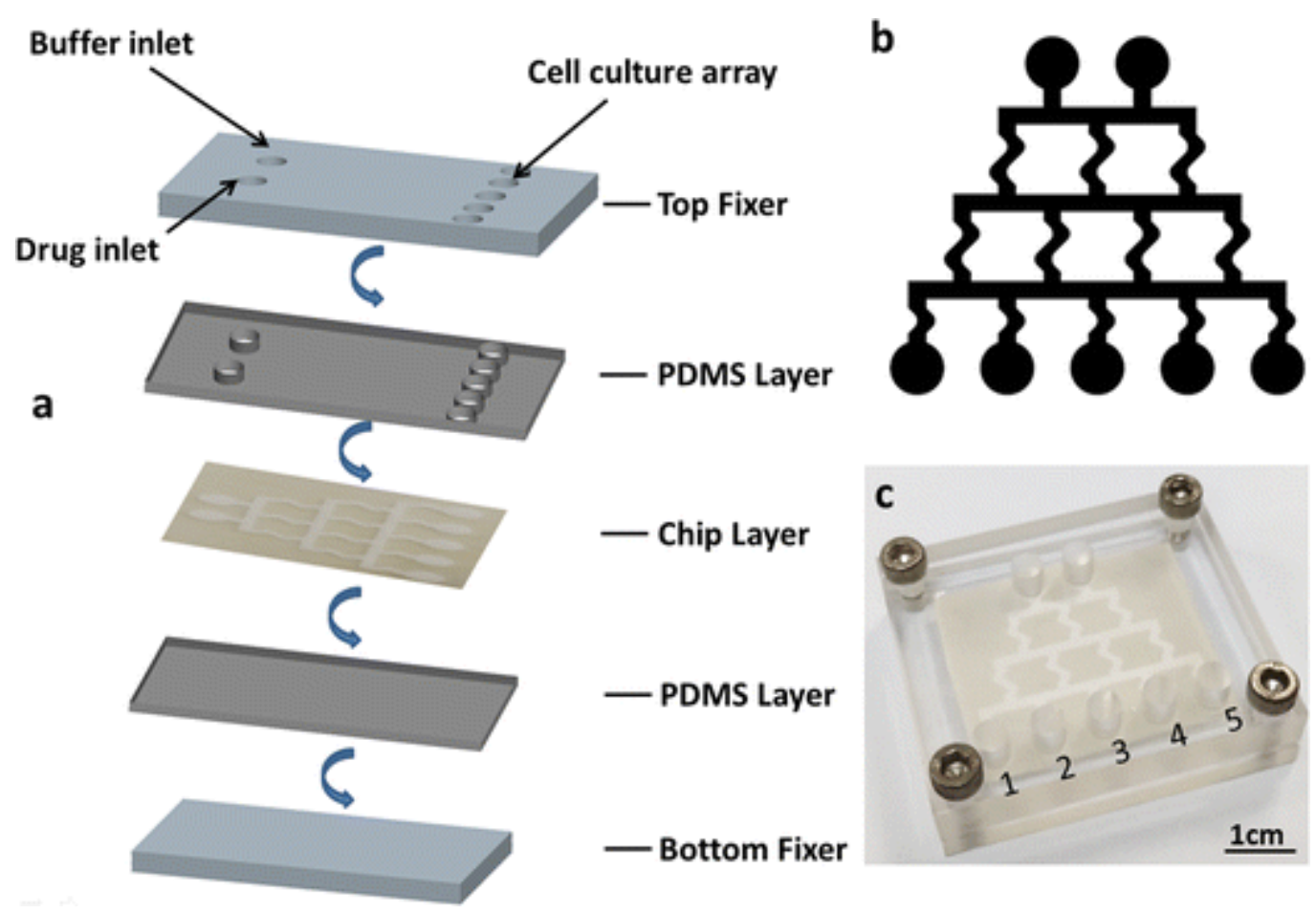

Fig. 6. Concentration gradient generator on a paper-based microfluidic chip coupled with cell culture microarray for high-throughput drug screening (Reprinted with permission from ref [106]). (a) Schematic illustration of device structure and assembly; (b) Design of paper channels for gradient generation; (c) Assembled device.

Apart from drug screening, paper-based microfluidic devices for drug delivery have also been reported. For example, Li et al developed an open-channel, water-in-oil emulsification in a paper microfluidic platform for drug delivery [107]. They presented novel open channel microfluidics based on surfaces with patterned wettability that had the ability to control the flow of virtually all high and low surface tension liquids (Fig. 7). The open channel devices were able to constrain various low surface tension oils at high enough flow rates to enable water-in-oil microfluidic emulsification in an open channel device. It could change the size of the emulsified droplets generated in the open channel device by adjusting the velocity of both the aqueous (dispersed) and organic (continuous) phases. Finally, they used a fabricated device to successfully synthesize relatively monodisperse, hydrogel microparticles that could incorporate a drug molecule. They also investigated the drug delivery characteristics of the fabricated particles. This open-channel microfluidic devices have the opportunity to achieve a high degree of fluid control with rapid and inexpensive fabrication. 
a

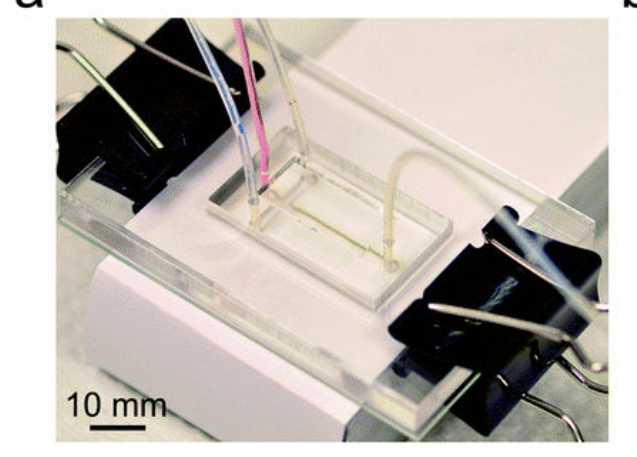

C

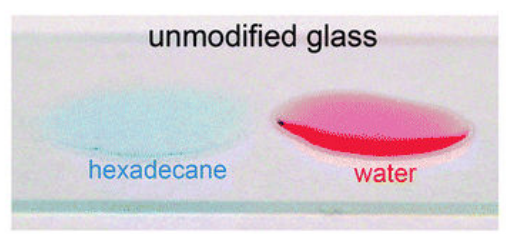

b

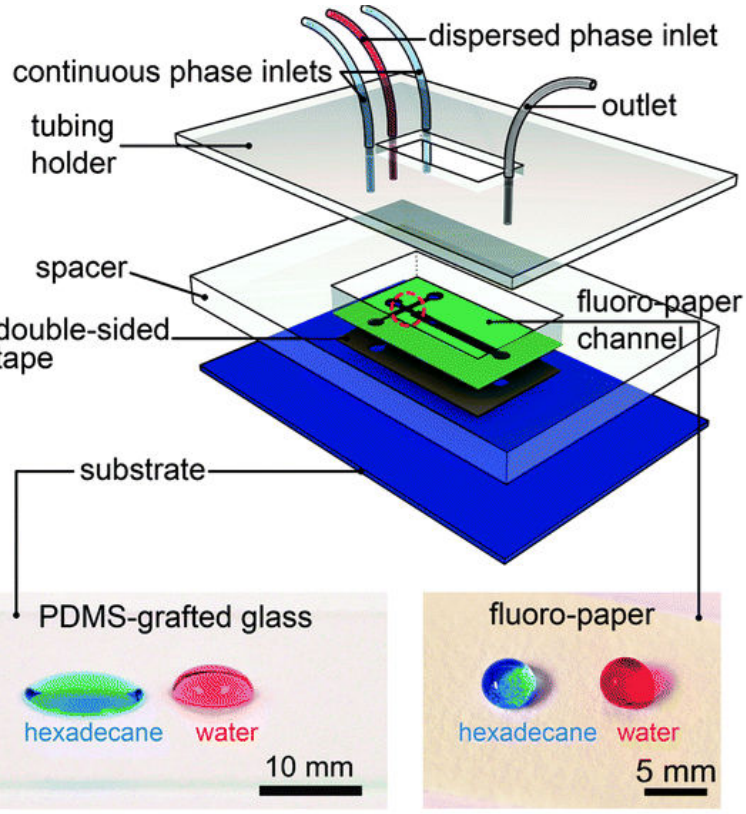

Fig. 7. An open-channel, water-in-oil emulsification in paper-based microfluidic devices for drug delivery (Reprinted with permission from ref [107]). (a) A photograph of the openchannel emulsification device. (b) A schematic of the emulsification device, (c) droplets of the hexadecane (blue) and water (red) on an unmodified glass substrate (far left), and on a substrate with a grafted PDMS thin film (middle).

Raphaël and Martin used a dynamic electrochemical quantitation of dopamine release from a cell-on-paper system [108]. They integrated an electrochemical method into a hybrid microfluidic system for the time-resolved detection of dopamine from neuron-like PC12 cells cultured on a patch of filter paper. They first investigated cell adherence to the surface of the paper with fluorescence microscopy. Then, dopamine release after stimulation with acetylcholine with or without drugs was studied. Those data obtained with the system were in agreement with single-cell data, thus demonstrating the validity of the method for the higher-throughput quantification of chemical targets on tissues- or organs-on-a-chip.

Overall, microfluidic devices hold many merits in the field of drug delivery, including the precise dosage, ideal delivery, target-precise delivery, sustainable and controlled release, multiple dosing, and slight side effects. These merits are important assets to drug delivery systems. Microfluidic techniques have been progressively used for the fabrication of drug carriers, direct drug delivery systems, high-throughput screening, and formulation and immobilization of drugs. Inexpensive and easily fabricated paper materials are rich substrates that naturally integrate several functionalities of transport, filtration, storage, concentrator, valving, and multiplexing. In the future, the paper microfluidic technology in controlled drug delivery applications would bring exciting opportunities to expand the body of knowledge in the field and promote the development of the clinical translation of drug delivery systems.

\section{Conclusion and perspectives}

As a highly sensitive and selective amplification technology for the detection of nucleic acids, LAMP has been widely employed for the rapid diagnosis of infectious diseases, analysis of food safety, diagnosis of veterinary and plant diseases and assessing 
environmental and public health concerns. The conventional analytical processes, including nucleic acid extraction, sample purification, and detection, could be incorporated into a microfluidic chip to construct a LAMP-based point-of-care assay. The combination of paper-based microfluidic devices and LAMP is one of the most powerful platforms for POC diagnostics. A critical factor of the current LAMP-based POC platform that needs to be considered is the feasibility and cost of the device in low-income settings. LAMP can be carried out without power as a paper microfluidic chip wicks aqueous fluids without the need for pumping or a power supply. Therefore, paper microfluidic device-based LAMP has great potential to become a cost-effective and efficient diagnostic tool used in geographically remote areas and low-income environments for POC analyses.

However, the loop primers of LAMP-based methods vigilantly need to be optimized for reproducible and sensitive target detection. Second, LAMP-based in vitro DNA amplification relies on polymerase, which is only licensed and supplied by several companies. Unless new universal enzymes for the LAMP strategy are constructed soon, this proprietary component would become a critical factor and limitation of a wide application of LAMP-based microfluidic devices. In addition, the stability of the enzyme and other chemicals also need to be taken into account. Biotechnologists must continue to study recombinant microorganisms and improve nucleic acid synthesizers to obtain more stable LAMP enzymes and other related reagents. New chemical processes and novel techniques need to be developed with nonenzymatic LAMP to be more useful for regions with limited resources. A wonderful LAMP-based on-site analysis needs to integrate upstream and downstream sample processes, such as sample preparation and nucleic acid extraction and amplification, with a sensitive and rapid sensing modality on a miniaturization microfluidic device for multiplex or even high-throughput detection of certain targets in a complicated sample. Smart integration is crucial to promote the development of LAMP-based devices. If upstream and downstream techniques are carefully optimized using multidisciplinary approaches, we believe that the LAMP-based paper devices as POC assays have great value for both academic and practical aspects due to its great merits, including low cost and ease of use.

A variety of microfluidic devices have been widely used for controlled drug delivery with rapid advances in micro- and nanotechnology [100]. These different microfluidic platforms have significantly promoted the development of the controlled drug delivery field in many different applications through the precise control of the drug delivery rate, time, and location and the increased efficacy with fewer side effects [10]. These microfluidic devices were fabricated and mainly focused on common materials, mostly from polydimethylsiloxane, glass, silicon, fused silica, hydrogel, and polytetrafluoroethylene [99]. Compared with other common materials, there are fewer reports about paper microfluidics used in drug delivery this far. Those reports on paper microfluidics used in drug screening and delivery reviewed by us showed unique advantages and have great potential in this field. Each material has its features regarding the ease and cost of device fabrication, the withstanding of high pressures and flow rates, and the resistance to organic solvents. As an abundant material, paper holds the functionalities of transport, filtration, storage, concentrator, and valving [16], and it has a large potential for the construction of paper-based microfluidics in the field of drug delivery, including in drug carrier-free micro-reservoir-based drug delivery systems, highly integrated drug carrier-free microfluidic systems, and drug carrier-integrated microfluidic systems. 
Despite the fact that much effort needs to be done in this area, we hope that paper microfluidics may serve as a proper candidate for a controlled drug delivery system. This will give clinically relevant drug formulations at desired kinetics to help effectively treat patients from different pathological conditions, reduce side effects, and increase the convenience of the patient.

\section{Acknowledgements}

This work is supported by the National Science Foundation of China-Project of Karst Science Research Center (U1612442), STS of Chinese Academy of Sciences (KFJ-STS-QYZD-185), Financial support from National Key Research and Development Program of China (No. 2019 YFC1803600), Science and Technology Program of Guizhou Province [Grant No. (2019)5618, 2019(2963), and (2019)4428], and Opening fund of State Key Laboratory of Environmental Geochemistry (SKLEG2019606, 2019717). ZY thanks UK NERC Fellowship grant (NE/R013349/1), SRPe grant (PECRE1819/05), Royal Academy of Engineering (FoDSF $\backslash 1819 \backslash 1 \backslash 8)$.

\section{References}

[1] J. Alonso-Padilla, N. Cortés-Serra, M.J. Pinazo, M.E. Bottazzi, M. Abril, F. Barreira, S. Sosa-Estani, P.J. Hotez, J. Gascón, Strategies to enhance access to diagnosis and treatment for Chagas disease patients in Latin America, Expert Rev. Anti-infe., 17 (2019) 145-157.

[2] M. Sharifi, F. Attar, A.A. Saboury, K. Akhtari, N. Hooshmand, A. Hasan, M.A. El-Sayed, M. Falahati, Plasmonic gold nanoparticles: Optical manipulation, imaging, drug delivery and therapy, J. Control. Release, 311-312 (2019) 170-189.

[3] L.Y. Yeo, C. Hsueh-Chia, P.P.Y. Chan, J.R. Friend, Microfluidic devices for bioapplications, Small, 7 (2011) 12-48.

[4] G. Du, Q. Fang, J.M.J. den Toonder, Microfluidics for cell-based high throughput screening platforms-A review, Anal. Chim. Acta, 903 (2016) 36-50.

[5] J.H. Tsui, W. Lee, S.H. Pun, J. Kim, D.-H. Kim, Microfluidics-assisted in vitro drug screening and carrier production, Adv. Drug. Deliver. Rev., 65 (2013) 1575-1588.

[6] C. Zhang, D. Xing, Single-Molecule DNA Amplification and Analysis Using Microfluidics, Chem. Rev., 110 (2010) 4910-4947.

[7] X. Chen, J. Shen, Review of membranes in microfluidics, J. Chem. Technol. Biot., 92(2017) 271-282.

[8] S.J. Han, H.K. Park, K.S. Kim, Applications of Microfluidic Devices for Urology, Int. Neurourol. J., 21 (2017) S4-9.

[9] R. McNerney, P. Daley, Towards a point-of-care test for active tuberculosis: obstacles and opportunities, Nat. Rev. Microbiol., 9 (2011) 204-213.

[10] E.I. Mancera-Andrade, A. Parsaeimehr, A. Arevalo-Gallegos, G. Ascencio-Favela, S.R. Parra, Microfluidics technology for drug delivery: A review, Front. Biosci., 10 (2018) 74.

[11] N.R. Harris, M. Hill, S. Beeby, Y. Shen, N.M. White, J.J. Hawkes, W.T. Coakley, A silicon microfluidic ultrasonic separator, Sensor. Actuat. B-Chem., 95 (2003) 425-434.

[12] W.H. Grover, A.M. Skelley, C.N. Liu, E.T. Lagally, R.A. Mathies, Monolithic membrane valves and diaphragm pumps for practical large-scale integration into glass microfluidic devices, Sensor. Actuat. B-Chem., 89 (2003) 315-323.

[13] X. Chen, T. Li, K. Zhai, Z. Hu, M. Zhou, Using orthogonal experimental method optimizing 
surface quality of $\mathrm{CO}_{2}$ laser cutting process for PMMA microchannels, Int. J. Adv. Manuf. Technol., 88 (2017) 2727-2733.

[14] H. Becker, L.E. Locascio, Polymer microfluidic devices, Talanta, 56 (2002) 267-287.

[15] L. Meng, Z. Deng, L. Niu, F. Li, F. Yan, J. Wu, F. Cai, H. Zheng, A Disposable Microfluidic Device for Controlled Drug Release from Thermal-Sensitive Liposomes by High Intensity Focused Ultrasound, Theranostics, 5 (2015) 1203-1213.

[16] N. Kaur, B.J. Toley, Paper-based nucleic acid amplification tests for point-of-care diagnostics, Analyst, 143 (2018) 2213-2234.

[17] Y. Yang, E. Noviana, M.P. Nguyen, B.J. Geiss, D.S. Dandy, C.S. Henry, Paper-Based Microfluidic Devices: Emerging Themes and Applications, Anal. Chem., 89 (2017) 71-91.

[18] M.M. Gong, D. Sinton, Turning the Page: Advancing Paper-Based Microfluidics for Broad Diagnostic Application, Chem. Rev., 117 (2017) 8447-8480.

[19] A.W. Martinez, S.T. Phillips, M.J. Butte, G.M. Whitesides, Patterned Paper as a Platform for Inexpensive, Low-Volume, Portable Bioassays, Angew. Chem. Edit., 119 (2007) 1340-1342.

[20] L. Magro, C. Escadafal, P. Garneret, B. Jacquelin, A. Kwasiborski, J.-C. Manuguerra, F. Monti, A. Sakuntabhai, J. Vanhomwegen, P. Lafaye, P. Tabeling, Paper microfluidics for nucleic acid amplification testing (NAAT) of infectious diseases, Lab Chip, 17 (2017) 2347-2371.

[21] J. Reboud, G.L. Xu, A. Garrett, M. Adriko, Z.G. Yang, E.M. Tukahebwa, C. Rowell, J.M. Cooper, Paper-based microfluidics for DNA diagnostics of malaria in low resource underserved rural communities, Proc. Natl. Acad. Sci. USA., 116 (2019) 4834-4842.

[22] P.T. Trieu, N.Y. Lee, Paper-Based All-in-One Origami Microdevice for Nucleic Acid Amplification Testing for Rapid Colorimetric Identification of Live Cells for Point-of-Care Testing, Anal. Chem., 91 (2019) 11013-11022.

[23] K. Mitsakakis, V. D'Acremont, S. Hin, F. von Stetten, R. Zengerle, Diagnostic tools for tackling febrile illness and enhancing patient management, Microelectron. Eng., 201 (2018) 26-59.

[24] M.S. Reid, X.C. Le, H. Zhang, Exponential Isothermal Amplification of Nucleic Acids and Assays for Proteins, Cells, Small Molecules, and Enzyme Activities: An EXPAR Example, Angew. Chem. Int. Edit., 57 (2018) 11856-11866.

[25] P. Gill, A. Ghaemi, Nucleic Acid Isothermal Amplification Technologies-A Review, Nucleos., Nucleot. Nucl., 27 (2008) 224-243.

[26] Y. Zhao, F. Chen, Q. Li, L. Wang, C. Fan, Isothermal Amplification of Nucleic Acids, Chem. Rev., 115 (2015) 12491.

[27] H. Qi, S. Yue, S. Bi, C. Ding, W. Song, Isothermal exponential amplification techniques: From basic principles to applications in electrochemical biosensors, Biosens. Bioelectron., 110 (2018) 207-217.

[28] C.-M. Chang, W.-H. Chang, C.-H. Wang, J.-H. Wang, J.D. Mai, G.-B. Lee, Nucleic acid amplification using microfluidic systems, Lab Chip, 13 (2013) 1225-1242.

[29] R. Duncan, E. Grigorenko, C. Fisher, D. Hockman, B. Lanning, Advances in multiplex nucleic acid diagnostics for blood-borne pathogens: promises and pitfalls - an update, Expert. Rev. Mol. Diagn., 19 (2019) 15-25.

[30] C.D. Ahrberg, A. Manz, B.G. Chung, Polymerase chain reaction in microfluidic devices, Lab Chip, 16 (2016) 3866.

[31] P.T. Monis, S. Giglio, Nucleic acid amplification-based techniques for pathogen detection and identification, Infection, Genet. Evol., 6 (2006) 2-12. 
[32] M.C. Giuffrida, G. Spoto, Integration of isothermal amplification methods in microfluidic devices: Recent advances, Biosens. Bioelectron., 90 (2017) 174-186.

[33] P.J. Asiello, A.J. Baeumner, Miniaturized isothermal nucleic acid amplification, a review, Lab Chip, 11 (2011) 1420-1430.

[34] T. Notomi, H. Okayama, H. Masubuchi, T. Yonekawa, K. Watanabe, N. Amino, T. Hase, Loop-mediated isothermal amplification of DNA, Nucleic Acids Res., 28 (2000) E63-E63.

[35] G.T. Walker, J.G. Nadeau, P.A. Spears, J.L. Schram, C.M. Nycz, D.D. Shank, Multiplex strand displacement amplification (SDA) and detection of DNA sequences from Mycobacterium tuberculosis and other mycobacteria, Nucleic Acids Res., 22 (1994) 2670-2677.

[36] P.M. Lizardi, X. Huang, Z. Zhu, P. Brayward, D.C. Thomas, D.C. Ward, Mutation detection and single-molecule counting using isothermal rolling-circle amplification, Nat. Genet., 19 (1998) 225-232.

[37] X. Zhang, S.B. Lowe, J.J. Gooding, Brief review of monitoring methods for loop-mediated isothermal amplification (LAMP), Biosens. Bioelectron., 61 (2014) 491-499.

[38] M.-H. Deng, L.-Y. Zhong, O. Kamolnetr, Y. Limpanont, Z.-Y. Lv, Detection of helminths by loop-mediated isothermal amplification assay: a review of updated technology and future outlook, Infect. Dis. Poverty, 8 (2019) 20.

[39] V.P. Dave, T.A. Ngo, A.-K. Pernestig, D. Tilevik, K. Kant, T. Nguyen, A. Wolff, D.D. Bang, MicroRNA amplification and detection technologies: opportunities and challenges for point of care diagnostics, Lab. Invest., 99 (2019) 452-469.

[40] J.R. Choi, J. Hu, Y. Gong, S. Feng, A.W. Wan, B. Pingguan-Murphy, F. Xu, An integrated lateral flow assay for effective DNA amplification and detection at the point of care, Analyst, 141 (2016) 2930.

[41] J.R. Choi, J. Hu, Y. Gong, S. Feng, W.A.B.W. Abas, B. Pingguan-Murphy, F. Xu, An integrated lateral flow assay for effective DNA amplification and detection at the point of care, Analyst, 141 (2016) 2930-2939.

[42] J. Hui, G. Yin, Y. Zhu, Y. Chen, S.J. Guo, S.C. Tao, Z. Yan, L. Peng, Multiplex sample-to-answer detection of bacteria using a pipette-actuated capillary array comb with integrated DNA extraction, isothermal amplification, and smartphone detection, Lab Chip, 18 (2018) 2854-2864.

[43] M. Safavieh, M.K. Kanakasabapathy, F. Tarlan, M.U. Ahmed, M. Zourob, W. Asghar, H. Shafiee, Emerging Loop-Mediated Isothermal Amplification-Based Microchip and Microdevice Technologies for Nucleic Acid Detection, ACS Biomater. Sci. Eng., 2 (2016) 278-294.

[44] H. Wang, Z. Ma, J. Qin, Z. Shen, Q. Liu, X. Chen, H. Wang, Z. An, W. Liu, M. Li, A versatile loop-mediated isothermal amplification microchip platform for Streptococcus pneumoniae and Mycoplasma pneumoniae testing at the point of care, Biosens. Bioelectron., 126 (2019) 373-380.

[45] X. Ye, J. Xu, L. Lu, X. Li, X. Fang, J. Kong, Equipment-free nucleic acid extraction and amplification on a simple paper disc for point-of-care diagnosis of rotavirus A, Anal. Chim. Acta, 1018 (2018) 78-85.

[46] T.N.D. Trinh, N.Y. Lee, A rapid and eco-friendly isothermal amplification microdevice for multiplex detection of foodborne pathogens, Lab Chip, 18 (2018) 2369-2377.

[47] D.M. Cate, J.A. Adkins, J. Mettakoonpitak, C.S. Henry, Recent Developments in Paper-Based Microfluidic Devices, Anal. Chem., 87 (2015) 19-41.

[48] A.M. López-Marzo, A. Merkoçi, Paper-based sensors and assays: a success of the engineering design and the convergence of knowledge areas, Lab Chip, 16 (2016) 3150-3176.

[49] Y. Xia, J. Si, Z. Li, Fabrication techniques for microfluidic paper-based analytical devices and 
their applications for biological testing: A review, Biosens. Bioelectron., 77 (2016) 774-789.

[50] A.W. Martinez, S.T. Phillips, G.M. Whitesides, E. Carrilho, Diagnostics for the Developing World: Microfluidic Paper-Based Analytical Devices, Anal. Chem., 82 (2010) 3-10.

[51] K. Macek, H. Bečváŕová, Papers, ready-for-use plates, and flexible sheets for chromatography, Chromatogr. Rev., 15 (1971) 1-28.

[52] R. Pelton, Bioactive paper provides a low-cost platform for diagnostics, TrAC-Trend. Anal. Chem., 28 (2009) 925-942.

[53] A. Apilux, W. Dungchai, W. Siangproh, N. Praphairaksit, C.S. Henry, O. Chailapakul, Lab-on-paper with dual electrochemical/colorimetric detection for simultaneous determination of gold and iron, Anal. Chem., 82 (2010) 1727.

[54] E. Carrilho, A.W. Martinez, G.M. Whitesides, Understanding Wax Printing: A Simple Micropatterning Process for Paper-Based Microfluidics, Anal. Chem., 81 (2009) 7091-7095.

[55] H. Li, A.J. Steckl, Paper Microfluidics for Point-of-Care Blood-Based Analysis and Diagnostics, Anal. Chem., 91 (2019) 352-371.

[56] N.A. Meredith, C. Quinn, D.M. Cate, J. Volckens, C.S. Henry, Paper-based analytical devices for environmental analysis, Analyst, 141 (2016) 1874-1887.

[57] A. Nilghaz, L. Guan, W. Tan, S. Wei, Advancement of Paper-based Microfluidics for Diagnostics the Original Motivation and Current status, ACS Sensors, 1 (2016) 1382-1393.

[58] Y. Yang, E. Noviana, M.P. Nguyen, B.J. Geiss, D.S. Dandy, C.S. Henry, Paper-Based Microfluidic Devices: Emerging Themes and Applications, Anal. Chem., 89 (2016) 71-91.

[59] J.-W. Chen, Y.Y. Lau, T. Krishnan, K.-G. Chan, C.-Y. Chang, Recent Advances in Molecular Diagnosis of Pseudomonasaeruginosa Infection by State-of-the-Art Genotyping Techniques, Front. Microbiol., 9 (2018) 1104-1104.

[60] J. Li, J. Macdonald, Advances in isothermal amplification: novel strategies inspired by biological processes, Biosens. Bioelectron., 64 (2015) 196-211.

[61] Z.-H. Cai, Y.-Y. Dai, L.-Y. Huang, W.-S. Zhang, X.-G. Guo, Diagnosis of mycoplasma pneumoniae by loop-mediated isothermal amplification: systematic review and meta-analysis, BMC Infect. Dis., 19 (2019) 173.

[62] K.P. Anupama, A. Chakraborty, I. Karunasagar, I. Karunasagar, B. Maiti, Loop-mediated isothermal amplification assay as a point-of-care diagnostic tool for Vibrio parahaemolyticus: recent developments and improvements, Exp. Rev. Mol. Diagn., 19 (2019) 229-239.

[63] N. Tomita, Y. Mori, H. Kanda, T. Notomi, Loop-mediated isothermal amplification (LAMP) of gene sequences and simple visual detection of products, Nat. Protoc., 3 (2008) 877-882.

[64] H. Zhang, Y. Xu, Z. Fohlerova, H. Chang, C. Iliescu, P. Neuzil, LAMP-on-a-chip: Revising microfluidic platforms for loop-mediated DNA amplification, TrAC Trend. Anal. Chem., 113 (2019) 44-53.

[65] G. Xu, D. Nolder, J. Reboud, M.C. Oguike, D.A. van Schalkwyk, C.J. Sutherland, J.M. Cooper, Paper-Origami-Based Multiplexed Malaria Diagnostics from Whole Blood, Angew. Chem. Int. Edit., 55 (2016) 15250-15253.

[66] K. Kaarj, P. Akarapipad, J.Y. Yoon, Simpler, Faster, and Sensitive Zika Virus Assay Using Smartphone Detection of Loop-mediated Isothermal Amplification on Paper Microfluidic Chips, Sci. Rep., 8 (2018) 12438-.

[67] W. Cevallos, P. Fernandez-Soto, M. Calvopina, M. Buendia-Sanchez, J. Lopez-Aban, B. Vicente, A. Muro, Diagnosis of amphimeriasis by LAMPhimerus assay in human stool samples long-term 
storage onto filter paper, PLoS One, 13 (2018).

[68] K. Pisamayarom, A. Suriyasomboon, P. Chaumpluk, Simple Screening of Listeria monocytogenes Based on a Fluorescence Assay via a Laminated Lab-On-Paper Chip, Biosens. Basel, 7 (2017).

[69] M. Safavieh, V. Kaul, S. Khetani, A. Singh, K. Dhingra, M.K. Kanakasabapathy, M.S. Draz, A. Memic, D.R. Kuritzkes, H. Shafiee, Paper microchip with a graphene-modified silver nano-composite electrode for electrical sensing of microbial pathogens, Nanoscale, 9 (2017) 1852-1861.

[70] P. Chaumpluk, P. Plubcharoensook, S. Prasongsuk, Rapid detection of aflatoxigenic Aspergillus sp in herbal specimens by a simple, bendable, paper-based lab-on-a-chip, Biotechnol. J., 11 (2016) 768-779.

[71] M. Dou, D.C. Dominguez, X. Li, J. Sanchez, G. Scott, A Versatile PDMS/Paper Hybrid Microfluidic Platform for Sensitive Infectious Disease Diagnosis, Anal. Chem., 86 (2014) 7978-7986.

[72] N.M. Rodriguez, W.S. Wong, L. Liu, R. Dewar, C.M. Klapperich, A fully integrated paperfluidic molecular diagnostic chip for the extraction, amplification, and detection of nucleic acids from clinical samples, Lab Chip, 16 (2016) 753-763.

[73] P. Chen, C. Chen, Y. Liu, W. Du, X. Feng, B.-F. Liu, Fully integrated nucleic acid pretreatment, amplification, and detection on a paper chip for identifying EGFR mutations in lung cancer cells, Sensor. Actuat. B-Chem., 283 (2019) 472-477.

[74] B. Li, X. Zhou, H. Liu, H. Deng, R. Huang, D. Xing, Simultaneous Detection of Antibiotic Resistance Genes on Paper-Based Chip Using Ru(phen)(2)dppz (2+) Turn-on Fluorescence Probe, ACS Appl. Mater. Inter., 10 (2018) 4494-4501.

[75] B.-H. Song, S.-I. Yun, M. Woolley, Y.-M. Lee, Zika virus: History, epidemiology, transmission, and clinical presentation, J. Neuroimmunol., 308 (2017) 50-64.

[76] S.A. Rasmussen, D.J. Jamieson, M.A. Honein, L.R. Petersen, Zika Virus and Birth Defects Reviewing the Evidence for Causality, New Engl. J. Med., 374 (2016) 1981-1987.

[77] Y. Lustig, D. Sofer, M. Hindiyeh, E. Mendelson, LAMP assay for specific detection of Asian and African lineage Zika virus: will it meet the expectations?, Ann. Transl. Med., 6 (2018) 53.

[78] M. Mauk, J. Song, H.H. Bau, R. Gross, F.D. Bushman, R.G. Collman, C. Liu, Miniaturized devices for point of care molecular detection of HIV, Lab Chip, 17 (2017) 382-394.

[79] J.T. Connelly, J.P. Rolland, G.M. Whitesides, "Paper Machine" for Molecular Diagnostics, Anal. Chem., 87 (2015) 7595-7601.

[80] J.R. Choi, J. Hu, R. Tang, Y. Gong, S. Feng, H. Ren, T. Wen, X. Li, W.A.B.W. Abas, B. Pingguan-Murphy, F. Xu, An integrated paper-based sample-to-answer biosensor for nucleic acid testing at the point of care, Lab Chip, 16 (2016) 611-621.

[81] T.N.D. Trinh, N.Y. Lee, A foldable isothermal amplification microdevice for fuchsin-based colorimetric detection of multiple foodborne pathogens, Lab Chip, 19 (2019) 1397-1405.

[82] T.N.D. Trinh, H.C. La, N.Y. Lee, Fully Integrated and Foldable Microdevice Encapsulated with Agarose for Long-Term Storage Potential for Point-of-Care Testing of Multiplex Foodborne Pathogens, ACS Sensors, (2019).

[83] P. Naik, S. Jaitpal, P. Shetty, D. Paul, An integrated one-step assay combining thermal lysis and loop-mediated isothermal DNA amplification (LAMP) in $30 \mathrm{~min}$ from E. coli and M. smegmatis cells on a paper substrate, Sensor. Actuat. B-Chem., 291 (2019) 74-80.

[84] B. Pang, K. Fu, Y. Liu, X. Ding, J. Hu, W. Wu, K. Xu, X. Song, J. Wang, Y. Mu, C. Zhao, J. Li, Development of a self-priming PDMS/paper hybrid microfluidic chip using mixed-dye-loaded loop-mediated isothermal amplification assay for multiplex foodborne pathogens detection, Anal. Chim. 
Acta, 1040 (2018) 81-89.

[85] S. Roy, N.F. Mohd-Naim, M. Safavieh, M.U. Ahmed, Colorimetric Nucleic Acid Detection on Paper Microchip Using Loop Mediated Isothermal Amplification and Crystal Violet Dye, ACS Sensors, 2 (2017) 1713-1720.

[86] P. Chaumpluk, A. Suriyasomboon, A Simple Paper-Based Lab-on-a-Chip for the Detection of a Highly Pathogenic Strain of Porcine Reproductive and Respiratory Syndrome Virus, Aust. J. Chem., 67 (2014) 1434-1440.

[87] J. Oyhenart, Direct detection of Tritrichomonas foetus in cattle genital fluid trough loop mediated isothermal amplification of elongation factor 1 alpha 1, Vet. Parasitol., 261 (2018) 67-72.

[88] Z. Yang, G. Xu, J. Reboud, S.A. Ali, G. Kaur, J. McGiven, N. Boby, P.K. Gupta, P. Chaudhuri, J.M. Cooper, Rapid Veterinary Diagnosis of Bovine Reproductive Infectious Diseases from Semen Using Paper-Origami DNA Microfluidics, ACS Sensors, 3 (2018) 403-409.

[89] S. Roy, I. Abd Rahman, M.U. Ahmed, Paper-based rapid detection of pork and chicken using LAMP-magnetic bead aggregates, Anal. Methods, 8 (2016) 2391-2399.

[90] J. Davison, Y. Bertheau, The Theory and Practice of European Traceability Regulations for GM Food and Feed, Cereal Food. World, 53 (2008) 186-196.

[91] R. Takabatake, Y. Kagiya, Y. Minegish, S. Futo, K. Soga, K. Nakamura, K. Kondo, J. Mano, K. Kitta, Rapid Screening Detection of Genetically Modified Crops by Loop-Mediated Isothermal Amplification with a Lateral Flow Dipstick, J. Agr. Food Chem., 66 (2018) 7839-7845.

[92] C. James, Global status of commercialized biotech/GM crops, (2004).

[93] F. Zhang, L. Wang, R. Wang, Y. Ying, J. Wu, Simple Screening Strategy with Only Water Bath Needed for the Identification of Insect-Resistant Genetically Modified Rice, Anal. Chem., 87 (2015) 1523-1526.

[94] D. Cyranoski, Chinese cities scan sewers for signs of illegal drug use, Nature, 559 (2018) 310-311.

[95] K. Mao, J. Ma, X. Li, Z. Yang, Rapid duplexed detection of illicit drugs in wastewater using gold nanoparticle conjugated aptamer sensors, Sci. Total Environ., 688 (2019) 771-779.

[96] P.M. Choi, B. Tscharke, S. Samanipour, W.D. Hall, C.E. Gartner, J.F. Mueller, K.V. Thomas, J.W. O'Brien, Social, demographic, and economic correlates of food and chemical consumption measured by wastewater-based epidemiology, P. Nat. Acad. Sci., (2019) 201910242.

[97] Z. Yang, B. Kasprzyk-Hordern, C.G. Frost, P. Estrela, K.V. Thomas, Community Sewage Sensors for Monitoring Public Health, Environ. Sci. Technol., 49 (2015) 5845-5846.

[98] Z. Yang, G. Xu, J. Reboud, B. Kasprzyk-Hordern, J.M. Cooper, Monitoring Genetic Population Biomarkers for Wastewater-Based Epidemiology, Anal. Chem., 89 (2017) 9941-9945.

[99] D. Liu, H. Zhang, F. Fontana, J.T. Hirvonen, H.A. Santos, Microfluidic-assisted fabrication of carriers for controlled drug delivery, Lab Chip, 17 (2017) 1856-1883.

[100] S.T. Sanjay, W. Zhou, M. Dou, H. Tavakoli, L. Ma, F. Xu, X. Li, Recent advances of controlled drug delivery using microfluidic platforms, Adv. Drug. Deliver. Rev., 128 (2018) 3-28.

[101] H. Chen, Z. Yu, S. Bai, H. Lu, D. Xu, C. Chen, D. Liu, Y. Zhu, Microfluidic models of physiological or pathological flow shear stress for cell biology, disease modeling and drug development, TrAC Trend. Anal. Chem., 117 (2019) 186-199.

[102] E.I. Mancera-Andrade, A. Parsaeimehr, A. Arevalo-Gallegos, G. Ascencio-Favela, R. Parra Saldivar, Microfluidics technology for drug delivery: A review, Front. Biosci., 10 (2018) 74-91.

[103] M. Brzezinski, M. Socka, B. Kost, Microfluidics for producing polylactide nanoparticles and microparticles and their drug delivery application, Polym. Int., 68 (2019) 997-1014. 
[104] F. He, M.J. Zhang, W. Wang, Q.W. Cai, Y.Y. Su, Z. Liu, Y. Faraj, X.J. Ju, R. Xie, L.Y. Chu, Designable Polymeric Microparticles from Droplet Microfluidics for Controlled Drug Release, Adv. Mater. Technol., 4 (2019) 23.

[105] Y. Ding, J. Li, W. Xiao, K. Xiao, J. Lee, U. Bhardwaj, Z. Zhu, P. Digiglio, G. Yang, K.S. Lam, T. Pan, Microfluidic-Enabled Print-to-Screen Platform for High-Throughput Screening of Combinatorial Chemotherapy, Anal. Chem., 87 (2015) 10166-10171.

[106] B. Hong, P. Xue, Y. Wu, J. Bao, Y.J. Chuah, Y. Kang, A concentration gradient generator on a paper-based microfluidic chip coupled with cell culture microarray for high-throughput drug screening, Biomed. Microdev., 18 (2016) 21.

[107] C. Li, M. Boban, A. Tuteja, Open-channel, water-in-oil emulsification in paper-based microfluidic devices, Lab Chip, 17 (2017) 1436-1441.

[108] R. Trouillon, M.A.M. Gijs, Dynamic electrochemical quantitation of dopamine release from a cells-on-paper system, RSC Adv., 6 (2016) 31069-31073. 